\title{
A POLYNOMIAL VARIANT OF A PROBLEM OF DIOPHANTUS AND ITS CONSEQUENCES
}

\author{
Alan Filipin AND Ana JuRAsić \\ University of Zagreb and University of Rijeka, Croatia
}

\begin{abstract}
In this paper we prove that every Diophantine quadruple in $\mathbb{R}[X]$ is regular. In other words, we prove that if $\{a, b, c, d\}$ is a set of four non-zero elements of $\mathbb{R}[X]$, not all constant, such that the product of any two of its distinct elements increased by 1 is a square of an element of $\mathbb{R}[X]$, then

$$
(a+b-c-d)^{2}=4(a b+1)(c d+1) .
$$

Some consequences of the above result are that for an arbitrary $n \in \mathbb{N}$ there does not exist a set of five non-zero elements from $\mathbb{Z}[X]$, which are not all constant, such that the product of any two of its distinct elements increased by $n$ is a square of an element of $\mathbb{Z}[X]$. Furthermore, there can exist such a set of four non-zero elements of $\mathbb{Z}[X]$ if and only if $n$ is a square.
\end{abstract}

\section{INTRODUCTION}

Diophantus of Alexandria ([5]) noted that the product of any two elements of the set $\left\{\frac{1}{16}, \frac{33}{16}, \frac{17}{4}, \frac{105}{16}\right\}$ increased by 1 is a square of rational number. A set consisting of $m$ positive integers (rational numbers) with the property that the product of any two of its elements increased by 1 is a square of an integer (rational number) is therefore called a (rational) Diophantine $m$-tuple. The first Diophantine quadruple, the set $\{1,3,8,120\}$, was found by Fermat.

Many generalizations of the original problem of Diophantus were also considered, for example by adding a fixed integer $n$ instead of 1 or considering the problem over domains other than $\mathbb{Z}$ or $\mathbb{Q}$. We have the following definition:

Definition 1.1. Let $m \geq 2$ and let $R$ be a commutative ring with 1 . Let $n \in R$ be a non-zero element and let $\left\{a_{1}, \ldots, a_{m}\right\}$ be a set of $m$ distinct

2010 Mathematics Subject Classification. 11D09,11D45.

Key words and phrases. Diophantine $m$-tuples, polynomials. 
non-zero elements from $R$ such that $a_{i} a_{j}+n$ is a square of an element of $R$ for $1 \leq i<j \leq m$. The set $\left\{a_{1}, \ldots, a_{m}\right\}$ is called a Diophantine $m$-tuple with the property $D(n)$ or simply a $D(n)$-m-tuple in $R$.

We also need the next two definitions (see [25]). A $D(n)$-triple $\{a, b, c\}$ in $R$ such that

$$
a b+n=r^{2}, a c+n=s^{2}, b c+n=t^{2},
$$

where $r, s, t \in R$, is called regular if

$$
(c-b-a)^{2}=4(a b+n) .
$$

Equation (1.2) is symmetric under permutations of $a, b, c$. From (1.2), using (1.1), we get

$$
\begin{gathered}
c=c_{ \pm}=a+b \pm 2 r, \\
a c_{ \pm}+n=(a \pm r)^{2}, b c_{ \pm}+n=(b \pm r)^{2} .
\end{gathered}
$$

A $D(n)$-quadruple $\{a, b, c, d\}$ in $R$ is called regular if

$$
n(d+c-a-b)^{2}=4(a b+n)(c d+n)
$$

or, equivalently, if

$$
d=d_{ \pm}=a+b+c+\frac{2}{n}(a b c \pm r s t) .
$$

Equation (1.5) is also symmetric under permutations of $a, b, c, d$.

It is interesting to find upper bounds for the number $m$ of elements of the sets described in Definition 1.1. Gibbs ([26]) found some rational Diophantine sextuples, e.g. $\left\{\frac{11}{192}, \frac{35}{192}, \frac{155}{27}, \frac{512}{27}, \frac{1235}{48}, \frac{180873}{16}\right\}$, but no upper bound for the size $m$ of a rational Diophantine $m$-tuple is known. Dujella et al. ([20]) proved that there exist infinitely many rational Diophantine sextuples. In the integer case, which is the most studied one, very recently He, Togbé and Ziegler ([27]) proved the folklore conjecture that there does not exist a Diophantine quintuple. There is also a stronger version of that conjecture which states that every Diophantine triple can be extended to a quadruple with a larger element in a unique way (see [11]):

Conjecture 1.2. If $\{a, b, c, d\}$ is a Diophantine quadruple and $d>$ $\max \{a, b, c\}$, then $d=d_{+}=a+b+c+2(a b c+\sqrt{(a b+1)(a c+1)(b c+1)})=$ $a+b+c+2(a b c+r s t)$.

This conjecture is still open. In 1979, Arkin, Hoggatt and Strauss ([1]) proved that every Diophantine triple $\{a, b, c\}$ can be extended to a Diophantine quadruple of integers $\left\{a, b, c, d_{+}\right\}$. Baker and Davenport ([2]) proved Conjecture 1.2 for the triple $\{a, b, c\}=\{1,3,8\}$ with the unique extension $d=d_{+}=120$. Many other results are also known (see $[4,7,22,24]$ ) which support this conjecture. 
Dujella $([9,10])$ found upper bounds for the size $m$ of $D(n)-m$-tuples in integer case. For similar results see $[12,13,18,26]$. Brown ([3]) proved that if $n$ is an integer, $n \equiv 2(\bmod 4)$, then there does not exist a $D(n)$ quadruple of integers. Furthermore, Dujella ([6]) proved that if $n$ is an integer satisfying $n \neq \equiv 2(\bmod 4)$ and $n \notin S=\{-4,-3,-1,3,5,8,12,20\}$, then there exists at least one $D(n)$-quadruple of integers, and if $n \notin S \cup T$, where $T=$ $\{-15,-12,-7,7,13,15,21,24,28,32,48,60,84\}$, then there exist at least two distinct $D(n)$-quadruples of integers. For some integers the question of the existence of such a quadruple is still open, as it is stated in Dujella's conjecture $([8])$ :

Conjecture 1.3. For $n \in S=\{-4,-3,-1,3,5,8,12,20\}$ there does not exist a $D(n)$-quadruple of integers.

The question of whether there exists a $D(n)$-quadruple can be reduced to elements of the set $S^{\prime}=\{-3,-1,3,5,8,20\}$ (see [6, Remark 3]).

In this paper we consider a polynomial variant of the problem, which was first studied by Jones $([28,29])$ for the case $R=\mathbb{Z}[X]$ and $n=1$. Also some other variants of such a polynomial problem were considered (see $[13,16,17,18,21,23])$. In the case where $R$ is a polynomial ring and $n$ is a constant polynomial, it is usually assumed that not all polynomials in such a $D(n)$-tuple are constant. In this paper we first consider the case where $R=\mathbb{R}[X]$ and $n=1$. To get other interesting results, we then apply the obtained result to $R=\mathbb{Z}[X]$ and $n$ is a positive integer. In particular, we prove a version of Conjecture 1.2 for Diophantine quadruples in $\mathbb{R}[X]$ :

TheOREM 1.4. Every $D(1)$-quadruple in $\mathbb{R}[X]$ is regular.

One easily sees that any $D(1)$-pair $\{a, b\}$ in $\mathbb{R}[X]$ can be extended to a regular $D(1)$-quadruple in $\mathbb{R}[X]$ :

$$
\{a, b, a+b+2 r, 4 r(a+r)(b+r)\},
$$

where $r$ is such that $a b+1=r^{2}$, as in (1.1). We note that Dujella and Fuchs ([13]) proved that every $D(1)$-quadruple in $\mathbb{Z}[X]$ is regular.

From $([18])$ it follows that there does not exist a $D(n)$-8-tuple in $\mathbb{Z}[X]$ for $n \in \mathbb{Z} \backslash\{0\}$. The right hand side of (1.5) is a square, so in $\mathbb{Z}[X]$ a regular $D(n)$-quadruple may exist only if $n$ is a perfect square, whereas regular $D(n)$ triples exist for every $n$. From (1.7), it follows that in $\mathbb{R}[X]$ a regular $D(n)$ quadruple ${ }^{1}$ exists for every positive integer $n$. Furthermore, in $\mathbb{C}[X]$ a regular $D(n)$-quadruple exists for every non-zero integer $n$.

An irregular $D(n)$-quadruple is one that is not regular. Suppose that $\{a, b, c, d\}$ is an irregular $D(n)$-quadruple in $\mathbb{Z}[X]$ for $n \in \mathbb{N}$. Then, the set $\left\{\frac{a}{\sqrt{n}}, \frac{b}{\sqrt{n}}, \frac{c}{\sqrt{n}}, \frac{d}{\sqrt{n}}\right\}$ would be an irregular $D(1)$-quadruple in $\mathbb{R}[X]$, which

\footnotetext{
${ }^{1}$ For example, the set $\left\{X, 4 X+4 \sqrt{5}, 9 X+6 \sqrt{5}, \frac{144}{5} X^{3}+48 \sqrt{5} X^{2}+124 X+20 \sqrt{5}\right\}$ is a regular $D(5)$-quadruple in $\mathbb{Q}(\sqrt{5})[X]$.
} 
contradicts Theorem 1.4. For every $n \in \mathbb{N}$ which is a perfect square a regular $D(n)$-quadruple in $\mathbb{Z}[X]$ can be obtained from the $D(1)$-quadruple (1.7) by multiplying its elements by $\sqrt{n}$. Since for $n \in \mathbb{N}$ which is not a perfect square there does not exist a regular $D(n)$-quadruple in $\mathbb{Z}[X]$, Theorem 1.4 yields:

COROLlary 1.5. There does not exist a $D(n)$-quadruple in $\mathbb{Z}[X]$ for any positive integer $n$ which is not a perfect square. Furthermore, there does not exist a $D(n)$-quintuple in $\mathbb{Z}[X]$ for any positive integer $n$.

Let us mention that there exists a $D(n)$-sextuple in $\mathbb{Z}[X]$, where $n$ is not a constant polynomial (see $[19,20]$ ). Moreover, in all those examples $n$ is a square in $\mathbb{Z}[X]$, while for $n \in \mathbb{Z}[X]$ non-square, there exist examples of $D(n)$-quintuples in $\mathbb{Z}[X]$ (see $[14]$ ).

Dujella and Fuchs ([12]) proved that there does not exist a $D(-1)$ quadruple in $\mathbb{Z}[X]$, i.e. they proved a polynomial variant of Conjecture 1.3 for $n=-1$. For an integer $n<0$ we cannot apply Theorem 1.4 to observe a $D(n)$ quadruple in $\mathbb{Z}[X]$ because in that case a $D(1)$-quadruple $\left\{\frac{a}{\sqrt{n}}, \frac{b}{\sqrt{n}}, \frac{c}{\sqrt{n}}, \frac{d}{\sqrt{n}}\right\}$ is from $\mathbb{C}[X]$. Using Corollary 1.5 we prove a special case of a polynomial variant of Conjecture 1.3:

Corollary 1.6. For $n \in\{3,5,8,12,20\}$ there does not exist a $D(n)$ quadruple in $\mathbb{Z}[X]$.

In order to prove Theorem 1.4 we partially follow the strategy used in [13] for $\mathbb{Z}[X]$ but we need to introduce some new ideas. In Section 2 we transform the problem of extending a $D(1)$-triple $\{a, b, c\}$ in $\mathbb{R}[X]$ to a $D(1)$-quadruple $\{a, b, c, d\}$ in $\mathbb{R}[X]$ into solving a system of simultaneous Pellian equations, which reduces the problem to finding intersections of binary recurring sequences of polynomials. In Section 3 we find a gap principle for the degrees of elements in a $D(1)$-triple $\{a, b, c\}$ in $\mathbb{R}[X]$. In Section 4 we describe all possible initial terms of the observed recurring sequences. We extend some results from [13] and use some results for $\mathbb{C}[X]$ from $[18]$. Hence, the problem is somewhat situated between analogue problems in $\mathbb{Z}[X]$ and $\mathbb{C}[X]$, that were studied before. Using results from Sections 2, 3 and 4, eventually in Section 5 we prove Theorem 1.4.

\section{Reduction to Intersections OF RECURSIVE SEQUENCES}

Let $\mathbb{R}^{+}[X]$ denote the set of all polynomials with real coefficients whose leading coefficient is positive. For $a, b \in \mathbb{R}[X]$, we say that $a<b$ if $b-a \in$ $\mathbb{R}^{+}[X]$. For $a \in \mathbb{R}[X]$, we define $|a|=a$ if $a \geq 0$ and $|a|=-a$ if $a<0$.

Let us consider an arbitrary extension of a $D(1)$-triple $\{a, b, c\}$ in $\mathbb{R}[X]$, where $a<b<c$, to a $D(1)$-quadruple $\{a, b, c, d\}$ in $\mathbb{R}[X]$. We first observe the equations

$$
a b+1=r^{2}, a c+1=s^{2}, b c+1=t^{2}
$$


for $r, s, t \in \mathbb{R}^{+}[X]$. Let $A, B, C, R, S, T$ be the leading coefficients of the polynomials $a, b, c, r, s, t$, respectively. By $(2.1)$, we have $A B=R^{2}, A C=S^{2}$ and $B C=T^{2}$. Hence, there is no loss of generality in assuming that $a, b, c \in$ $\mathbb{R}^{+}[X]$. By $[21$, Lemma 1$]$, there is at most one constant polynomial in a $D(1)$ tuple in $\mathbb{C}[X]$. Let us denote by $\alpha, \beta, \gamma$ the degrees of $a, b, c$, respectively. We will use this notation throughout the paper. Hence, $0 \leq \alpha \leq \beta \leq \gamma$ and $\beta, \gamma>0$. Let

$$
a d+1=x^{2}, \quad b d+1=y^{2}, \quad c d+1=z^{2},
$$

where $x, y, z \in \mathbb{R}[X]$. Note that $x, y$ and $z$ can be $<0$, which is relevant because otherwise by taking only positive values we would exclude some possibilities we have in $\mathbb{C}[X]$. By $(2.2)$, we have $d \in \mathbb{R}^{+}[X]$.

Eliminating $d$ from (2.2), we obtain the system of simultaneous Pellian equations

$$
\begin{aligned}
& a z^{2}-c x^{2}=a-c, \\
& b z^{2}-c y^{2}=b-c .
\end{aligned}
$$

We want to find solutions $(z, x)$ and $(z, y)$ of (2.3) and (2.4), respectively.

Lemma 2.1. Let $(z, x)$ and $(z, y)$ be solutions, with $x, y, z \in \mathbb{R}[X]$, of (2.3) and (2.4), respectively. Then there exist solutions $\left(z_{0}, x_{0}\right)$ and $\left(z_{1}, y_{1}\right)$, with $z_{0}, x_{0}, z_{1}, y_{1} \in \mathbb{R}[X]$, of (2.3) and (2.4), respectively, such that:

$$
\begin{array}{ll}
\left|z_{0}\right| \geq 1, \quad & \left|x_{0}\right| \geq 1, \\
\left|z_{1}\right| \geq 1, & \left|y_{1}\right| \geq 1
\end{array}
$$

and

$$
\begin{array}{rlrl}
\operatorname{deg}\left(z_{0}\right) & \leq \frac{3 \gamma-\alpha}{4}, & \operatorname{deg}\left(x_{0}\right) \leq \frac{\alpha+\gamma}{4}, \\
\operatorname{deg}\left(z_{1}\right) \leq \frac{3 \gamma-\beta}{4}, & \operatorname{deg}\left(y_{1}\right) \leq \frac{\beta+\gamma}{4} .
\end{array}
$$

There also exist non-negative integers $m$ and $n$ such that

$$
\begin{gathered}
z \sqrt{a}+x \sqrt{c}=\left(z_{0} \sqrt{a}+x_{0} \sqrt{c}\right)(s+\sqrt{a c})^{m}, \\
z \sqrt{b}+y \sqrt{c}=\left(z_{1} \sqrt{b}+y_{1} \sqrt{c}\right)(t+\sqrt{b c})^{n} .
\end{gathered}
$$
4].

Proof. The statements (2.7) - (2.10) follow directly from [21, Lemma

From the proof of $[21$, Lemma $4(\mathrm{v})]$, we have that if $c \mid\left(z^{2}-1\right)$, then $c \mid\left(z_{0}^{2}-1\right)$. Hence, there exists $d_{0} \in \mathbb{R}[X]$ such that $c d_{0}=z_{0}^{2}-1$. Then, by (2.3), we have $a d_{0}=x_{0}^{2}-1$. Therefore,

$$
a d_{0}+1=x_{0}^{2} \text { and } c d_{0}+1=z_{0}^{2} .
$$


Since $c>0$, it follows from (2.11) that $\operatorname{deg}\left(z_{0}\right)>0$ for $d_{0} \neq 0$. Thus, $d_{0} \geq 0$. Furthermore, by (2.11), we obtain $x_{0}^{2} \geq 1$ and $z_{0}^{2} \geq 1$, so (2.5) holds. The proof of (2.6) is analogous. We consider $d_{1} \in \mathbb{R}[X]$, where

$$
b d_{1}+1=y_{1}^{2}, \quad c d_{1}+1=z_{1}^{2} .
$$

From (2.12), we see that $d_{1} \geq 0$.

By (2.9) and (2.10) of Lemma 2.1, we get $z=v_{m}=w_{n}$ for some $(m, n)$, where the sequences $\left(v_{m}\right)_{m \geq 0}$ and $\left(w_{n}\right)_{n \geq 0}$ are defined by

$$
\begin{aligned}
v_{0}=z_{0}, & v_{1}=s z_{0}+c x_{0}, \quad v_{m+2}=2 s v_{m+1}-v_{m}, \\
w_{0}=z_{1}, & w_{1}=t z_{1}+c y_{1}, \quad w_{n+2}=2 t w_{n+1}-w_{n},
\end{aligned}
$$

where the initial values $\left(z_{0}, x_{0}\right)$ and $\left(z_{1}, y_{1}\right)$ satisfy the estimates $(2.7)$ and (2.8), respectively. Hence, we reduced the problem of finding extensions of $D(1)$-triple $\{a, b, c\}$ to solving the equation

$$
v_{m}=w_{n}
$$

in $m, n \geq 0$.

REMARK 2.2. We remark that we cannot assume $z_{0}>0$ or $z_{0}<0$ since we would lose some solutions of the Pellian equation (2.3). The same holds for $z_{1}$. But, without loss of generality we may assume that $x_{0}>0$ because, by (2.2), for $z$ and $-z$ we obtain the same $d$. The analogue situation is for $y_{1}$.

By [21, Lemma 5], for $m \geq 1$ we have

$$
\operatorname{deg}\left(v_{m}\right)=(m-1) \frac{\alpha+\gamma}{2}+\operatorname{deg}\left(v_{1}\right) .
$$

Also, by [21, Lemma 5], (2.1), (2.7) and (2.13), we obtain

$$
\frac{\gamma}{2} \leq \operatorname{deg}\left(v_{1}\right) \leq \frac{\alpha+5 \gamma}{4}
$$

Similarly, for $n \geq 1$,

$$
\operatorname{deg}\left(w_{n}\right)=(n-1) \frac{\beta+\gamma}{2}+\operatorname{deg}\left(w_{1}\right)
$$

and, by (2.1), (2.8) and (2.14), we get

$$
\frac{\gamma}{2} \leq \operatorname{deg}\left(w_{1}\right) \leq \frac{\beta+5 \gamma}{4}
$$




\section{GAP PRINCIPLE FOR DEGREES}

In this section we give a gap principle describing all possible relations between the degrees $\alpha, \beta$ and $\gamma$ of the elements of the triple $\{a, b, c\}$ in $\mathbb{R}[X]$. We need the following identities proved for elements of $\mathbb{Z}[X]$ (see $[16,30]$ ), which also hold in $\mathbb{R}[X]$. Recall that $r, s, t \in \mathbb{R}^{+}[X]$ are polynomials satisfying $a b+1=r^{2}, a c+1=s^{2}$ and $b c+1=t^{2}$. Also, recall that we reduced the problem of finding extensions of $D(1)$-triple $\{a, b, c\}$ to solving the equation $v_{m}=w_{n}$, where the sequences $\left(v_{m}\right)_{m \geq 0}$ and $\left(w_{n}\right)_{n \geq 0}$ are given by

$$
\begin{aligned}
& v_{0}=z_{0}, v_{1}=s z_{0}+c x_{0}, v_{m+2}=2 s v_{m+1}-v_{m}, \\
& w_{0}=z_{1}, w_{1}=t z_{1}+c y_{1}, w_{n+2}=2 t w_{n+1}-w_{n} .
\end{aligned}
$$

LEMmA 3.1. Let $\{a, b, c\}$, where $a<b<c$, be a $D(1)$-triple in $\mathbb{R}[X]$ such that (2.1) holds. Then for

$$
d_{ \pm}=a+b+c+2(a b c \pm r s t)
$$

we have

$$
a d_{ \pm}+1=u_{ \pm}^{2}, b d_{ \pm}+1=v_{ \pm}^{2}, c d_{ \pm}+1=w_{ \pm}^{2}
$$

where

$$
u_{ \pm}=a t \pm r s, v_{ \pm}=b s \pm r t, w_{ \pm}=c r \pm s t .
$$

Furthermore, we have

$$
c=a+b+d_{ \pm}+2\left(a b d_{ \pm} \mp r u_{ \pm} v_{ \pm}\right)
$$

and

$$
c=a+b-d_{ \pm}+2 r w_{ \pm} .
$$

Proof. By [16, Lemma 1], relations (3.2) and (3.3) are true.

From the proof of [16, Lemma 3], we have $u_{-}, v_{-}<0$. Obviously, $u_{+}, v_{+}>0$. By (3.4), we have $c=e_{\mp}$, where $e_{\mp}$ is obtained by applying (3.1) on the $D(1)$-triple $\left\{a, b, d_{ \pm}\right\}$. For $d_{-}=0$, using (3.2) and (3.4), we obtain $c=c_{+}$. Otherwise, by (3.2), we have $d_{-}>0$. By (3.4) with the lower signs, we conclude that $c>a+b$, so $c^{2}>c(a+b)+1$. By that and $(2.1)$, we have $s^{2} t^{2}=a b c^{2}+c(a+b)+1<c^{2} r^{2}$. Therefore, $w_{-}=c r-s t>0$. Obviously, $w_{+}>0$. From (3.4), using (2.1) and (3.3), we get (3.5).

REMARK 3.2. In the proof of Lemma 3.1 we saw that $w_{ \pm}>0$. This will be quite important in the following considerations.

By (3.1), we conclude

$$
\operatorname{deg}\left(d_{+}\right)=\alpha+\beta+\gamma>\gamma .
$$

For $d_{-} \neq 0$, from [15, Lemma 1] and [18, Lemma 2], we have $c>2 a b d_{-}$. Thus, $0 \leq \operatorname{deg}\left(d_{-}\right) \leq \gamma-\alpha-\beta$, in particular $\gamma \geq \alpha+\beta$. But, we are able to prove even more: 
Lemma 3.3. Let $\{a, b, c\}$ be a $D(1)$-triple in $\mathbb{R}[X]$, where $a<b<c$, and let $d_{-}$be defined by (3.1). Then $d_{-}=0$ or $\operatorname{deg}\left(d_{-}\right)=\gamma-\alpha-\beta$.

Proof. Let $d_{-} \neq 0$. By (2.1) and (3.2), we have $\operatorname{deg}\left(a b d_{-}\right)=\alpha+\beta+$ $\operatorname{deg}\left(d_{-}\right)=\operatorname{deg}\left(r u_{-} v_{-}\right)$. If $\beta<\gamma$, by (3.4), we get $\operatorname{deg}\left(d_{-}\right)=\gamma-\alpha-\beta$.

Let $\beta=\gamma$. Since $\operatorname{deg}\left(a b d_{-}\right) \geq \gamma$, we have $a b d_{-}>0$ and $r u_{-} v_{-}>0$. Then, by (3.4), we obtain $\operatorname{deg}\left(a b d_{-}\right)=\gamma$ and $-\alpha=\operatorname{deg}\left(d_{-}\right)=0$. We cannot have two different constants in a $D(1)$-quadruple in $\mathbb{R}[X]$, so if $\beta=\gamma$ then $d_{-}=0$ or $d_{-}=a$.

A $D(1)$-quadruple with the property from Definition 1.1, but with a relaxed condition that its elements need not be distinct ${ }^{2}$ and need not be nonzero is called improper $D(1)$-quadruple. Such a quadruple can be regular or irregular the same way as defined before. In $\mathbb{R}[X]$ there always exist improper $D(1)$-quadruples $\{0, a, b, c\}$ and also $\{a, a, b, c\}$ if $a$ is a constant ${ }^{3}$. Also, there always exist regular $D(1)$-quadruples $\left\{a, b, c, d_{ \pm}\right\}$. Hence, the equation (2.15) always has solutions.

Assume that $\left\{a, b, c, d^{\prime}\right\}$, where $a<b<c$, with $\operatorname{deg}\left(d^{\prime}\right)=\delta$ and $\gamma \leq \delta$, is an irregular $D(1)$-quadruple with minimal possible $\delta$ among all irregular $D(1)$-quadruples in $\mathbb{R}[X]$. We prove that such a quadruple does not exist. By $[18$, Lemma 5], we have

$$
\delta \geq \frac{3 \beta+5 \gamma}{2}
$$

Lemma 3.4. Let $\{a, b, c\}$, with $a<b<c$, be a $D(1)$-triple in $\mathbb{R}[X]$. Let $d_{-}$be defined by (3.1). Assume that (2.15) holds and define $d=\frac{v_{m}^{2}-1}{c}$.

a) If $d=d_{-}$, then $v_{m}=w_{n}= \pm w_{-}$with $m, n \in\{0,1\}$.

b) If $d=d^{\prime}$, then $v_{m}=w_{n}= \pm z$ with $m \geq 3$ and $n \geq 3$.

c) If $0 \in\{m, n\}$, then $d=d_{-}$or $d=0 \neq d_{-}$or $d=a \neq d_{-}$and $a \in \mathbb{R}^{+}$.

d) If $m=n=1$, then $d=d_{-}$or $d=0 \neq d_{-}$or $d=a \neq d_{-}$and $a \in \mathbb{R}^{+}$ or $d=d_{+}$and $\gamma \geq \alpha+2 \beta$.

Proof. a) If $d_{-}=0$, then $w_{-}=1, \operatorname{so} \operatorname{deg}\left(w_{-}\right)=0$. By Lemma 3.3 and (3.2), if $d_{-} \neq 0$, then

$$
\operatorname{deg}\left(w_{-}\right)=\gamma-\frac{\alpha+\beta}{2}<\gamma
$$

By (2.16) and (2.18), we have $\operatorname{deg}\left(v_{m}\right) \geq \gamma$ for $m \geq 2$ and $\operatorname{deg}\left(w_{n}\right) \geq \gamma$ for $n \geq 2$. Thus, $d_{-}$must arise from $v_{m}=w_{n}$ for $m, n \in\{0,1\}$.

b) If $d=d^{\prime}$, by [18, Proposition 1], we have $m \geq 3$ and $n \geq 3$.

\footnotetext{
${ }^{2}$ In an improper $D(1)$-quadruple we cannot have two equal non-constant polynomials because the equation $b^{2}+1=t^{2}$ has no solution $t \in \mathbb{R}[X]$ for every non-constant $b \in \mathbb{R}[X]$.

${ }^{3}$ The equation $a^{2}+1=u_{-}^{2}$ has a solution $u_{-} \in \mathbb{R}$ for every $a \in \mathbb{R}$. This does not hold in $\mathbb{Z}$ for $a \neq 0$. In $\mathbb{Q}$ it does hold for some values of $a$.
} 
c) If $0 \in\{m, n\}$ then, by the proof of [18, Proposition 1], we have $\operatorname{deg}(d)<$ $\gamma$. By Lemma 3.3, we can have $d=d_{-}$. By (3.6), $d \neq d_{+}$. By (3.7) and the minimality assumption, the only possible irregular quadruples $\{a, b, c, d\}$ are those with $d=0$ or $d=a$ if $a \in \mathbb{R}^{+}$.

d) Let $v_{1}=w_{1}$. By the proof of [18, Proposition 1], $\operatorname{deg}(d)<\gamma$ and $\{a, b, c, d\}$ is an irregular quadruple (by the minimality assumption, then $d=0$ or $d=a$ if $a \in \mathbb{R}^{+}$), or $d=d_{ \pm}$. By (3.2) and (3.6), we have $\operatorname{deg}\left(w_{+}\right)=\gamma+\frac{\alpha+\beta}{2}$. Hence, by (2.17), we can have $d=d_{+}$and $w_{+}=v_{1}$ if $\gamma \geq \alpha+2 \beta$.

In the following lemma we consider all possibilities for $d_{-}$. That will give us all possible relations between degrees of the polynomials in our $D(1)$ triple. A similar gap principle is well known in the classical case and was also used in considering a polynomial variants of the problem of Diophantus (see e.g. [13, Lemma 4]), but we give more information about the possible triples. Conclusions about degrees generally hold, but conclusions about initial terms hold only for the case a) of Lemma 3.4, which means that we consider only such equations (2.15) from which the solution $d=d_{-}$arises.

Lemma 3.5. Let $\{a, b, c\}$ be a $D(1)$-triple in $\mathbb{R}[X]$, with $a<b<c$, for which (2.1) holds. Let $d_{-}$be defined by (3.1). We have:

1.) If $d_{-}=0$, then $z_{0}=z_{1}= \pm 1$. In this case $c=a+b+2 r$ and $\beta=\gamma$. Also, if $\alpha<\beta$, then $C=B$, and if $\alpha=\beta$, then $C=A+B+2 \sqrt{A B}$.

2.) a) If $d_{-}=a \in \mathbb{R}^{+}$, then $\left(z_{0}, z_{1}\right)=( \pm s, \pm s), \alpha=0, \beta=\gamma$ and $c=b+2 r s$.

b) If $d_{-} \in \mathbb{R}^{+} \backslash\{a\}$, then $z_{0}=z_{1}= \pm c r \mp s t, \alpha>0$ and $\gamma=\alpha+\beta$.

3.) If $\operatorname{deg}\left(d_{-}\right)>0$, then we have the following possibilities:

a) $z_{0}=z_{1}= \pm c r \mp$ st, with $\operatorname{deg}\left(d_{-}\right) \leq \alpha, \alpha>0$ and $\alpha+\beta<\gamma \leq$ $2 \alpha+\beta$

b) $\left(z_{0}, z_{1}\right)=($ cr - st, $-s)$, where $\alpha \leq \operatorname{deg}\left(d_{-}\right) \leq \beta, \alpha \geq 0$ and $2 \alpha+\beta \leq \gamma \leq \alpha+2 \beta$

c) $\left(z_{0}, z_{1}\right)=(-t, c r-s t)$, with $\operatorname{deg}\left(d_{-}\right)=\alpha, \alpha=\beta$ and $\gamma=3 \alpha$,

d) $\left(z_{0}, z_{1}\right)=(-t,-s)$, where $\beta \leq \operatorname{deg}\left(d_{-}\right)<\gamma, \alpha \geq 0$ and $\gamma \geq$ $\alpha+2 \beta$.

Proof. 1.) From (3.4), if $d_{-}=0$, then the triple $\{a, b, c\}$ is regular. By (1.3), we have $\gamma \leq \beta$, thus $\gamma=\beta$. Also, if $\alpha<\beta$, then $C=B$, and if $\alpha=\beta$ then $C=A+B+2 \sqrt{A B}$.

By (3.2), we have $w_{-}=1$. By (2.17) and (2.19), we conclude $\operatorname{deg}\left(v_{1}\right)$, $\operatorname{deg}\left(w_{1}\right) \geq \frac{\gamma}{2}$, so $v_{0}=w_{0}= \pm 1$. By (2.13), (2.14) and Remark 2.2, we get $z_{0}=z_{1}= \pm 1$.

2.) If $\operatorname{deg}\left(d_{-}\right)=0$ then, by Lemma 3.3 , we have

$$
\gamma=\alpha+\beta .
$$


2.a) Let $d_{-}=a \in \mathbb{R}_{+}$. By (3.9), we get $\beta=\gamma$. By (3.2), we have $w_{-}=s$, so by (3.5) we conclude

$$
c=b+2 r s .
$$

By Lemma $3.4 \mathrm{a}$ ), we have $v_{m}=w_{n}= \pm s$ for $m, n \in\{0,1\}$. For $m=$ $n=0$, by (2.13) and (2.14), we obtain $z_{0}=z_{1}= \pm s$. For $(m, n)=(0,1)$, by (2.13) and (2.14), we get

$$
z_{0}=t z_{1}+c y_{1}= \pm s .
$$

From (2.12) and (3.11), we have $\pm c r \mp s t=t z_{1}+c y_{1}$. Since $\operatorname{deg}(c r+s t)>\gamma$ and, by $(2.7), \operatorname{deg}\left(z_{0}\right)<\gamma$, other combinations of the signs on the left hand side of the previous equation are not possible. Hence,

$$
c\left( \pm r-y_{1}\right)=t\left(z_{1} \pm s\right) .
$$

By (2.1), we conclude $t \mid\left( \pm r-y_{1}\right)$. By considering degrees of polynomials, we obtain that $y_{1}= \pm r$ and $z_{1}=\mp s$. From Remark 2.2, we have $y_{1}=r$. By Lemma 3.1, cr $-s t>0$, thus, (3.11) implies $z_{0}=s$ and $z_{1}=-s$. For $(m, n)=(1,0)$, by $(2.13)$ and $(2.14), s z_{0}+c x_{0}=z_{1}= \pm s$. Hence, $c x_{0}=$ $s\left( \pm 1-z_{0}\right)$. By $(2.1), s \mid x_{0}$. Since $x_{0} \neq 0$, this is not possible ${ }^{4}$ because of the degrees of $s$ and $x_{0}$. For $(m, n)=(1,1)$, by (2.13) and $(2.14)$, we have $s z_{0}+c x_{0}=t z_{1}+c y_{1}= \pm s$. As for $(m, n)=(1,0)$, this is not possible.

2.b) Assume now that we have $d_{-} \in \mathbb{R}^{+} \backslash\{a\}$. From (3.9), since we cannot have two different constants in a $D(1)$-quadruple, $\alpha>0$. By (3.3), $w_{-}=c r-s t \neq s$. By Lemma $\left.3.4 \mathrm{a}\right)$, we have $v_{m}=w_{n}= \pm c r \mp s t$ for $m, n \in$ $\{0,1\}$. For $(m, n)=(0,0)$, by (2.13) and (2.14), we have $z_{0}=z_{1}= \pm c r \mp s t$. For $(m, n)=(0,1)$, by $(2.13)$ and $(2.14)$, we get

$$
z_{0}=t z_{1}+c y_{1}= \pm c r \mp s t .
$$

We obtain (3.12), so again $y_{1}=r$ and $z_{1}=-s$. Using (2.12) and (3.9), from $(2.8)$, we obtain a contradiction. Analogously as before, the cases $(m, n)=$ $(1,0)$ and $(m, n)=(1,1)$ are not possible.

3. If $\operatorname{deg}\left(d_{-}\right)>0$, then, by Lemma 3.3, we have $\gamma>\alpha+\beta$. By Lemma 3.4 a), $v_{m}=w_{n}= \pm c r \mp s t$ for $m, n \in\{0,1\}$.

3.a) For the case $(m, n)=(0,0)$, by $(2.13)$ and $(2.14)$, we have $z_{0}=z_{1}=$ $\pm c r \mp$ st. Using (3.8) and (2.8), we obtain $\gamma \leq 2 \alpha+\beta$. Lemma 3.3 implies $\operatorname{deg}\left(d_{-}\right) \leq \alpha$. Hence, $\alpha>0$.

3.b) For the case $(m, n)=(0,1)$, by (2.13) and (2.14), we have (3.13) and then (3.12). As in 2.a), $y_{1}=r, z_{1}=-s$ and $z_{0}=c r-s t$. By (3.8) and (2.7), $\gamma \leq \alpha+2 \beta$. By Lemma 3.3, $\operatorname{deg}\left(d_{-}\right) \leq \beta$. Using (2.1) and (2.8), we get $\gamma \geq 2 \alpha+\beta$ thus, by Lemma 3.3, $\operatorname{deg}\left(d_{-}\right) \geq \alpha$. The case where $\alpha=0$ and $d_{-}=a$ is considered in 2.a).

\footnotetext{
${ }^{4} \operatorname{In} \mathbb{C}[X]$ for $x_{0}=0$ we have $a= \pm i$ and $z_{0}^{2}= \pm c i+1$.
} 
3.c) For the case $(m, n)=(1,0)$, similarly as in 3.b), we get $x_{0}=r$, $z_{0}=-t$ and $z_{1}=c r-s t$. Also, we obtain $\alpha+2 \beta \leq \gamma \leq 2 \alpha+\beta$ from which it follows that $\alpha=\beta$ and then $\gamma=3 \alpha$.

3.d) For the case $(m, n)=(1,1)$ we use results from 3.b) and 3.c). They lead to $z_{0}=-t$ and $z_{1}=-s$. The conclusion about the degrees follows from (2.7) and (2.8). By Lemma 3.3, we have $\operatorname{deg}\left(d_{-}\right) \geq \beta$.

It is not hard to find the examples ${ }^{5}$ of $D(1)$-triples for all cases from Lemma 3.5, so all cases are really possible.

REMARK 3.6. The case 2.a) of Lemma 3.5 can be described more precisely. By $(3.2), v_{-}=-r$ and $u_{-}^{2}=a^{2}+1$. From (3.4), we get

$$
c=b+2 r^{2}\left(a-u_{-}\right) .
$$

From (3.4), using (2.1) and (3.2), we also get

$$
c=b\left(a-u_{-}\right)^{2}+2\left(a-u_{-}\right) .
$$

By (3.3), we have $-r=b s-r t$, so

$$
r(t-1)=b s .
$$

By $(2.1), \operatorname{gcd}(b, r)=1$, so $r=p s$, where $p \in \mathbb{R}^{+}$. Since $b<c$ and $\beta=\gamma$, we have $B \leq C$. By comparing the leading coefficients in (3.16), we get $p=\frac{\sqrt{B}}{\sqrt{C}}$, so $0<p<1$ (for $p=1$ we would have $b=c$ ). Using (2.1), (3.14) and (3.2), since $u_{-}<0$, we furthermore conclude that $a-u_{-}=\frac{1}{p}$, i.e. $p=-u_{-}-a$. By (3.14), we obtain

$$
c=b+\frac{2}{p} r^{2}=b+2 p s^{2}
$$

\footnotetext{
${ }^{5}$ Examples: 1.) $\quad\left\{1, X^{2}+2 X, X^{2}+4 X+3\right\}$ and $\left.\{X-1, X+1,4 X\}, 2 . \mathrm{a}\right)$ $\left.\left\{\frac{4}{3}, \frac{4 X^{2}+2 X-2}{3}, 12 X^{2}+6 X\right\} ; d_{-}=\frac{4}{3}, \quad 2 . b\right)\left\{125 X^{2}+50 X, 12500000000 X^{10}+\right.$ $26000000000 X^{9}+23070000000 X^{8}+11392000000 X^{7}+3424950000 X^{6}+644520000 X^{5}+$ $75187000 X^{4}+5200000 X^{3}+194525 X^{2}+3300 X+15,1250000000000 X^{12}+$ $3100000000000 X^{11}+3372000000000 X^{10}+2114000000000 X^{9}+844190000000 X^{8}+$ $224024000000 X^{7}+40005200000 X^{6}+4764480000 X^{5}+367264500 X^{4}+17315400 X^{3}+$ $\left.452640 X^{2}+5500 X+\frac{96}{5}\right\} ; d_{-}=\frac{1}{5}$, 3.a) $\left\{16 X^{3}-4 X, 64 X^{5}-48 X^{3}+8 X, 4096 X^{9}+\right.$ $\left.4096 X^{8}-4096 X^{7}-4096 X^{6}+1408 X^{5}+1280 X^{4}-192 X^{3}-128 X^{2}+9 X+3\right\}$; $\left.d_{-}=X+1, \quad 3 . b\right)\left\{\frac{1}{5}, 12500000000 X^{10}+26000000000 X^{9}+23070000000 X^{8}+\right.$ $11392000000 X^{7}+3424950000 X^{6}+644520000 X^{5}+75187000 X^{4}+5200000 X^{3}+$ $194525 X^{2}+3300 X+15,1250000000000 X^{12}+3100000000000 X^{11}+3372000000000 X^{10}+$ $2114000000000 X^{9}+844190000000 X^{8}+224024000000 X^{7}+40005200000 X^{6}+$ $\left.4764480000 X^{5}+367264500 X^{4}+17315400 X^{3}+452640 X^{2}+5500 X+\frac{96}{5}\right\}$; $\left.\left.d_{-}=125 X^{2}+50 X, 3 . c\right) \quad\left\{X-1, X+1,16 X^{3}-4 X\right\} ; \quad d_{-}=4 X, \quad 3 . \mathrm{d}\right)$ $\left\{\frac{1}{5}, \frac{625 X^{2}+250 X}{5}, 12500000000 X^{10}+26000000000 X^{9}+23070000000 X^{8}+11392000000 X^{7}+\right.$ $\left.3424950000 X^{6}+644520000 X^{5}+75187000 X^{4}+5200000 X^{3}+194525 X^{2}+3300 X+15\right\}$; $d_{-}=125000000 X^{8}+210000000 X^{7}+144200000 X^{6}+52040000 X^{5}++10562000 X^{4}+$ $1195600 X^{3}+70160 X^{2}+1800 X+56 / 5$.
} 
Also, from (3.16), we have

$$
t=\frac{b}{p}+1
$$

From (3.17), using (3.18), we obtain

$$
c\left(\frac{1}{p}-2 a\right)=t+1 .
$$

From (3.18), (3.19) and (2.1), we obtain

$$
t+1=c p .
$$

By (3.18), (3.19) and (3.20), the triple from the case 2.a) of Lemma 3.5 has the form

$$
\{a, b, c\}=\left\{\frac{1-p^{2}}{2 p}, t p-p, \frac{t}{p}+\frac{1}{p}\right\} .
$$

Also, by (3.18),

$$
\{a, b, c\}=\left\{\frac{1-p^{2}}{2 p}, b, \frac{b}{p^{2}}+\frac{2}{p}\right\} .
$$
$\mathbb{R}[X]$.

In the following lemma, we adjust [13, Lemma 10] to the situation in

Lemma 3.7. Let $\{a, b, c\}$, where $a<b<c$, be a $D(1)$-triple in $\mathbb{R}[X]$ with $\beta<\gamma=\alpha+2 \beta$. Let $d_{-}$be defined by (3.1). Then $\left\{a, b, d_{-}, c\right\}$ has elements

$$
\begin{aligned}
& \left\{ \pm \frac{D_{-}-B}{2 \sqrt{B D_{-}}}, b, b \frac{D_{-}}{B} \pm 2 \frac{\sqrt{D_{-}}}{\sqrt{B}}\right. \\
& \left.\quad \pm 2 b^{2} \frac{\sqrt{D_{-}}}{\sqrt{B}}\left(\frac{D_{-}}{B}-1\right)+2 b\left(3 \frac{D_{-}}{B}-1\right) \pm \frac{9 D_{-}-B}{2 \sqrt{B D_{-}}}\right\}
\end{aligned}
$$

where $D_{-}$is the leading coefficient of $d_{-}$and the upper combination of the signs is for the case $b<d_{-}$, while the lower is for the case $b>d_{-}$.

Proof. For the triple $\{a, b, c\}$, by Lemma 3.3, we have $\operatorname{deg}\left(d_{-}\right)=\beta$. Hence, the triple $\left\{a, b, d_{-}\right\}$has the form 1.) or 2.a) from Lemma 3.5.

If the triple $\left\{a, b, d_{-}\right\}$is regular, by Definition 1.1, $d_{-}=a+b \pm 2 r$. Similarly as in [13, Lemma 10], $c=4 r(r \pm a)(b \pm r)$ and $s=2 r^{2} \pm 2 a r-1$.

Assume that the triple $\left\{a, b, d_{-}\right\}$has the form (3.22). Then $\alpha=0$ and $b<d_{-}$. Let us denote $p_{1}:=\frac{\sqrt{B}}{\sqrt{D_{-}}}$. By $(3.22), d_{-}=\frac{b}{p_{1}^{2}}+\frac{2}{p_{1}}$. By (3.18), we have $v_{-}=-\frac{b}{p_{1}}-1$ and we also have $u_{-}=-\frac{r}{p_{1}}$ (we use the fact that $\left.u_{-}, v_{-}<0\right)$. Using (2.1) and (3.2), from (3.4), we obtain $c=e_{+}$. From that, by applying (3.1) for the triple $\left\{a, b, d_{-}\right\}$and using the expression for $a$ from 
(3.22), we obtain $c=2 b^{2} \frac{1}{p_{1}}\left(\frac{1}{p_{1}^{2}}-1\right)+2 b\left(\frac{3}{p_{1}^{2}}-1\right)+\frac{9-p_{1}^{2}}{2 p_{1}}$. Hence, we have (3.24). Similarly, for $d_{-}<b$, we denote $p_{2}:=\frac{\sqrt{D-}}{\sqrt{B}}$ and we obtain $d_{-}=b p_{2}^{2}-2 p_{2}$, $v_{-}=-b p_{2}+1$ and $u_{-}=-p_{2} r$. By (3.1), for the triple $\left\{a, d_{-}, b\right\}$, we get $c=2 b^{2} p_{2}\left(1-p_{2}^{2}\right)+2 b\left(-1+3 p_{2}^{2}\right)+\frac{1-9 p_{2}^{2}}{2 p_{2}}$. Moreover, from (3.3) for that triple, we obtain $s=-a v_{-}-r u_{-}$. It implies that

$$
s= \pm\left(\frac{D_{-}}{B}-1\right) b+\frac{3 D_{-}-B}{2 \sqrt{B D_{-}}} .
$$

\section{Precise determination of initial terms}

In this section we will determine all possible initial terms of the recurring sequences $\left(v_{m}\right)_{m \geq 0}$ and $\left(w_{n}\right)_{n \geq 0}$ which lead to the extension of the $D(1)$-triple $\{a, b, c\}$, with $a<b<c$, in $\mathbb{R}[X]$. Recall that we are solving the equation $z=v_{m}=w_{n}$ where $z^{2}=c d+1$. In the rest of the paper we distinguish the cases of the equation (2.15) depending on the parity of indices $m$ and $n$. From (2.13) and (2.14), by induction, congruence relations from the following lemma hold for $m, n \geq 0$ (see [13]).

Lemma 4.1. Let the sequences $\left(v_{m}\right)$ and $\left(w_{n}\right)$ be given by (2.13) and (2.14). Then

$$
\begin{array}{ll}
v_{2 m} \equiv z_{0}(\bmod c), & v_{2 m+1} \equiv s z_{0}(\bmod c), \\
w_{2 n} \equiv z_{1}(\bmod c), & w_{2 n+1} \equiv t z_{1}(\bmod c) .
\end{array}
$$

The following lemma is a version for $\mathbb{R}[X]$ of $[18$, Lemma 3$]$, where Dujella and the second author described all possible relations between the initial terms $z_{0}$ and $z_{1}$ of the recurring sequences $\left(v_{m}\right)_{m \geq 0}$ and $\left(w_{n}\right)_{n \geq 0}$ in $\mathbb{C}[X]$.

Lemma 4.2. 1) If $v_{2 m}=w_{2 n}$, then $z_{0}=z_{1}$.

2) If $v_{2 m+1}=w_{2 n}$, then either $\left(z_{0}, z_{1}\right)=( \pm 1, \pm s)$ or $\left(z_{0}, z_{1}\right)=( \pm s, \pm 1)$ or $z_{1}=s z_{0} \pm c x_{0}$, where $x_{0}$ is not constant.

3) If $v_{2 m}=w_{2 n+1}$, then either $\left(z_{0}, z_{1}\right)=( \pm t, \pm 1)$ or $\left(z_{0}, z_{1}\right)=(\mp s, \pm 1)$ or $\left(z_{0}, z_{1}\right)=( \pm 1, \pm 1)$ or $z_{0}=t z_{1} \pm c y_{1}$, where $y_{1}$ is not constant.

4) If $v_{2 m+1}=w_{2 n+1}$, then either $\left(z_{0}, z_{1}\right)=(\mp 1, \pm c r \mp$ st $)$ or $\left(z_{0}, z_{1}\right)=$ $( \pm c r \mp s t, \mp 1)$ or $s z_{0} \pm c x_{0}=t z_{1} \pm c y_{1}$, where $x_{0}$ and $y_{1}$ are not constant and polynomials on both sides of the equation have degree less than $\gamma$.

Proof. The proof of part 1) is completely analogue to the proof of [18, Lemma 3,(1)].

2) We follow the proof of [18, Lemma 3, (2)]. From Lemma 4.1, we have $z_{1} \equiv s z_{0}(\bmod c)$. This congruence allows us to conclude that for the cases $\left(z_{0}, z_{1}\right)=( \pm 1, \pm s)$ and $\left(z_{0}, z_{1}\right)=( \pm s, \pm 1)$ we have $z_{0} z_{1}>0$. Otherwise we would have $c \mid s$ and $c \mid 2$, respectively. 
3) From Lemma 4.1, we have $z_{0} \equiv t z_{1}(\bmod c)$. Assume that $z_{1}= \pm 1$. Hence,

$$
z_{0} \equiv \pm t(\bmod c)
$$

If $\beta<\gamma$, then $z_{0}= \pm t$ and $z_{0} z_{1}>0$. If $\beta=\gamma$ then, from Lemma 3.3, it follows that $d_{-}=0$ or $d_{-}=a$. If $d_{-}=0$, then $c=a+b+2 r$ and we have $s \equiv-t(\bmod c)$. Multiplying this congruence by $s$, we obtain $1 \equiv-s t(\bmod c)$. Now, multiplying (4.1) by $s$, we get

$$
s z_{0} \pm c x_{0} \equiv \mp 1(\bmod c) \text {. }
$$

From $(4.1), z_{0} \equiv \mp s(\bmod c)$. If $\alpha<\gamma$, then $z_{0}=\mp s$, with $z_{0} z_{1}<0$. Let $\alpha=\beta=\gamma$. For $z_{0}= \pm 1$, similarly as in the proof of [18, Lemma 3, (2)], we obtain a contradiction. We conclude that $z_{0} \neq \pm 1$. Now, from [21, Lemma $5], \operatorname{deg}\left(z_{0}\right) \geq \frac{\gamma}{2}$ and $\operatorname{deg}\left(x_{0}\right) \geq \frac{\alpha}{2}$. If $\alpha=0$, we have $\beta=\gamma=0$, which is not possible. Hence, $x_{0}$ is not constant. Using (2.3), we get

$$
\left(s z_{0}+c x_{0}\right)\left(s z_{0}-c x_{0}\right)=s^{2} z_{0}^{2}-c^{2} x_{0}^{2}=z_{0}^{2}+a c-c^{2},
$$

from which we conclude that one of the polynomials $s z_{0} \pm c x_{0}$ has degree less than $\gamma$. For that polynomial, (4.2) becomes an equation. Note that $\operatorname{deg}\left(z_{0}\right) \leq \frac{3 \gamma-\alpha}{4}=\frac{\gamma}{2}$, so $\operatorname{deg}\left(z_{0}\right)=\frac{\gamma}{2}$. From [21, Lemma 4] it follows that $\operatorname{deg}\left(v_{0}\right)=\operatorname{deg}\left(z_{0}\right) \leq \operatorname{deg}\left(s z_{0} \pm c x_{0}\right)$, so we have a contradiction. For $d_{-}=a$ and $\alpha=0$, from $(3.20)$ and $(4.1)$, we have $z_{0} \equiv \pm 1(\bmod c)$. Therefore, $z_{0}= \pm 1$, with $z_{0} z_{1}>0$.

Assume now that $z_{1} \neq \pm 1$. By [21, Lemma 5], we have $\operatorname{deg}\left(z_{1}\right) \geq \frac{\gamma}{2}$ and $\operatorname{deg}\left(y_{1}\right) \geq \frac{\beta}{2}$. Hence, $y_{1}$ is not constant. Using (2.4), we get

$$
\left(c y_{1}+t z_{1}\right)\left(c y_{1}-t z_{1}\right)=c^{2} y_{1}^{2}-t^{2} z_{1}^{2}=c^{2}-b c-z_{1}^{2},
$$

so we conclude that one of the polynomials $t z_{1} \pm c y_{1}$ has degree less than $\gamma$ and they are both congruent to $z_{0}$ modulo $c$. Hence, one of these polynomials is equal to $z_{0}$.

4) We use the proof of [18, Lemma 3, (4)]. For $z_{0}= \pm 1$, because of (2.8), we have $\pm s t \mp c r=z_{1}$. Similarly, for $z_{1}= \pm 1$, because of $(2.7), \pm s t \mp c r=z_{0}$.

In $\mathbb{Z}[X]$ (see $[13$, Lemma 5$]$ ), if the equation (2.15) has a solution then there exists a solution with $m, n \in\{0,1\}$. In $\mathbb{R}[X]$ in those cases, by Lemma $3.4, d=d_{-}$or $d=d_{+}$and $\gamma \geq \alpha+2 \beta$ or $d=0 \neq d_{-}$or $d=a \neq d_{-}$, where $a \in \mathbb{R}^{+}$(which is not possible in $\mathbb{Z}[X]$ ). But there are also other possibilities from Lemma 4.2. We examine all of them, precisely determining all possible initial terms and give some additional information which hold in $\mathbb{R}[X]$.

LEMMA 4.3. 1) If $v_{2 m}=w_{2 n}$, then either

a) $z_{0}=z_{1}= \pm 1$ or

b) $z_{0}=z_{1}= \pm s$ and $\alpha=0$ or 
c) $z_{0}=z_{1}= \pm c r \mp$ st and $\alpha>0, \alpha+\beta \leq \gamma \leq 2 \alpha+\beta$.

2) If $v_{2 m+1}=w_{2 n}$, then either
a) $\left(z_{0}, z_{1}\right)=( \pm 1, \pm s)$ and $\gamma \geq 2 \alpha+\beta$ or
b) $\left(z_{0}, z_{1}\right)=( \pm s, \pm 1)$ and $\alpha=0$ or
c) $\left(z_{0}, z_{1}\right)=(-t, c r-s t)$ and $\alpha=\beta, \gamma=3 \alpha$.

3) If $v_{2 m}=w_{2 n+1}$, then either

a) $\left(z_{0}, z_{1}\right)=( \pm t, \pm 1)$ and $\gamma \geq \alpha+2 \beta$ or

b) $\left(z_{0}, z_{1}\right)=(\mp s, \pm 1)$ and $\alpha=0, \beta=\gamma$ or

c) $\left(z_{0}, z_{1}\right)=( \pm 1, \pm 1)$ and $\alpha=0, \beta=\gamma$ or

d) $\left(z_{0}, z_{1}\right)=(c r-s t,-s)$ and $\alpha \geq 0,2 \alpha+\beta \leq \gamma \leq \alpha+2 \beta$ (special case:

4) If $v_{2 m+1}=w_{2 n+1}$, then either

1) $\left(z_{0}, z_{1}\right)=(s,-s)$ and $\left.\alpha=0, \beta=\gamma\right)$.
a) $\left(z_{0}, z_{1}\right)=( \pm 1, \mp c r \pm s t)$ and $\gamma \leq 2 \alpha+\beta$ (special cases:
1) $\left(z_{0}, z_{1}\right)=( \pm 1, \mp 1)$ and $\alpha \leq \beta=\gamma$ and
2) $\left(z_{0}, z_{1}\right)=( \pm 1, \mp s)$ and $\left.\alpha=0, \beta=\gamma\right)$ or
b) $\left(z_{0}, z_{1}\right)=( \pm c r \mp s t, \mp 1)$ and $\gamma \leq \alpha+2 \beta$ (special cases:
1) 4.a.1) and
2) $\left(z_{0}, z_{1}\right)=( \pm s, \mp 1)$ and $\left.\alpha=0, \beta=\gamma\right)$ or
c) $\left(z_{0}, z_{1}\right)=( \pm t, \pm s)$ and $\gamma \geq \alpha+2 \beta$.

Proof. 1) From Lemma 4.2, $z_{0}=z_{1}$. By (2.13) and (2.14), we have $v_{0}=w_{0}$, thus we apply Lemma $3.4 \mathrm{c}$ ). The cases where $d=d_{-}$are described in 1.)-3.a) of Lemma 3.5. If $d=0 \neq d_{-}$, then $z_{0}=z_{1}= \pm 1$. By Lemma 3.3, if $\beta=\gamma$, then $d_{-}=a$ and $\alpha=0$. Otherwise, $\beta<\gamma$. If $d=a \neq d_{-}$and $\alpha=0$, then $z_{0}=z_{1}= \pm s$. By Lemma 3.3, if $\beta=\gamma$, then $d_{-}=0$. Otherwise, $\beta<\gamma$. Hence, we get the cases 1.a)-1.c).

2.) a) From Lemma 4.2 , we can have $\left(z_{0}, z_{1}\right)=( \pm 1, \pm s)$ with $z_{0} z_{1}>0$. Using (2.8), we have $\gamma \geq 2 \alpha+\beta$.

b) By Lemma 4.2 , we have $\left(z_{0}, z_{1}\right)=( \pm s, \pm 1)$ with $z_{0} z_{1}>0$. By $(2.7)$, we get $\gamma \geq 3 \alpha$. From (2.11), $x_{0}^{2}=a^{2}+1$ with $a \in \mathbb{R}^{+}$since otherwise the equation is not possible.

c) By Lemma 4.2, there is also a possibility $z_{1}=s z_{0} \pm c x_{0}$, where $x_{0}$ is not constant. By (2.8), (4.3) and Remark 2.2, we conclude that $s z_{0}-c x_{0}=z_{1}$, where $z_{0}>0$ and $z_{1}<0$, or $s z_{0}+c x_{0}=z_{1}$, where $z_{0}<0$ and $z_{1}>0$. That is $v_{1}=w_{0}$, thus, by Lemma $\left.3.4 \mathrm{c}\right), d_{1}=d_{-}$or $d_{1}=0 \neq d_{-}$or $d_{1}=a \neq d_{-}$ and $\alpha=0$. The case where $d_{1}=d_{-}$is described in 3.c) of Lemma 3.5. If $d_{1}=0 \neq d_{-}$, then $s z_{0} \pm c x_{0}= \pm 1$. If $\alpha=\gamma$, then, by Lemma 3.3, we get $\gamma=0$, which is a contradiction. Therefore, $\alpha<\gamma$. By $(4.3), \operatorname{deg}\left(s z_{0} \mp c x_{0}\right)=2 \gamma$, which is not possible because of (2.7). If $d_{1}=a \neq d_{-}$and $\alpha=0$, then $s z_{0} \pm c x_{0}= \pm s$. Here $s \mid x_{0}$, which is not possible since $x_{0} \neq 0$ and because of (2.7).

3.) a) This case is completely analogous to $2 . \mathrm{a}$ ). 
b) By Lemma 4.2, it can further be $\left(z_{0}, z_{1}\right)=(\mp s, \pm 1)$ with $z_{0} z_{1}<0$ and $\beta=\gamma$. Similarly as in 2.b), $a \in \mathbb{R}^{+}$.

c) From Lemma 4.2 , there is $\left(z_{0}, z_{1}\right)=( \pm 1, \pm 1)$, with $z_{0} z_{1}>0, \alpha=0$ and $\beta=\gamma$.

d) Finally, by Lemma 4.2 , there is a possibility $z_{0}=t z_{1} \pm c y_{1}$, where $y_{1}$ is not constant. By (2.7) and (4.4), we conclude that $z_{0}=t z_{1}-c y_{1}$, where $z_{0}<0$ and $z_{1}>0$, or $z_{0}=t z_{1}+c y_{1}$, where $z_{0}>0$ and $z_{1}<0$. Here we have $v_{0}=w_{1}$, so, by Lemma $3.4 \mathrm{c}$ ), we get $d_{0}=d_{-}$or $d_{0}=0 \neq d_{-}$or $d_{0}=a \neq d_{-}$and $a \in \mathbb{R}^{+}$. The cases where $d_{0}=d_{-}$are described in 2.a) and 3.b) of Lemma 3.5. Further, for the simplicity, we can observe 2.a) as the special case of 3.b). If $d_{0}=0 \neq d_{-}$, then $t z_{1} \pm c y_{1}= \pm 1$. If $\beta=\gamma$, then, by Lemma $3.3, d_{-}=a$, $\alpha=0$ and (3.10) holds. Hence, by (4.4), $\operatorname{deg}\left(t z_{1} \mp c y_{1}\right)=2 \gamma$. For $\beta<\gamma$, by (4.4), we also have $\operatorname{deg}\left(t z_{1} \mp c y_{1}\right)=2 \gamma$. This is not possible because of (2.8). If $d_{0}=a \neq d_{-}$and $\alpha=0$, then $t z_{1} \pm c y_{1}= \pm s$. If $\beta<\gamma$, then by (4.4), $\operatorname{deg}\left(t z_{1} \mp c y_{1}\right)=\frac{3 \gamma}{2}$, which is in contradiction with (2.8). If $\beta=\gamma$, then, by Lemma $3.3, d_{-}=0$ so $c=a+b+2 r$. Furthermore, by (1.3) and (4.4), $\operatorname{deg}\left(t z_{1} \mp c y_{1}\right)=\gamma$. By Lemma 4.1 , we have $t z_{1} \equiv \pm s(\bmod c)$. Multiplying that by $t$, we furthermore obtain $z_{1} \equiv \pm s t \mp c r(\bmod c)$. Since

$$
( \pm s t-c r)( \pm s t+c r)=a c+b c+1-c^{2}
$$

one of the polynomials $\pm s t \mp c r$ has degree less then $\gamma$ and the other has degree equal to $\gamma+\frac{\alpha+\beta}{2}$. Hence, $\pm s t \mp c r=z_{1}$. By (1.3) and (4.5), we have $\operatorname{deg}\left(z_{1}\right)=0$. Then, $z_{1}=\mp 1$, which implies $y_{1}=1$ and that is not possible.

4.) a) By Lemma 4.2, we firstly can have $\left(z_{0}, z_{1}\right)=( \pm 1, \mp c r \pm s t)$, i.e. $\left(z_{0}, z_{1}\right)=\left( \pm 1, \mp w_{-}\right)$. By $(2.8)$, we get the bound for $\gamma$. Specially, for $d_{-}=0$, by Lemma 3.3 and $(3.2), z_{1}=\mp 1$ and $\alpha \leq \beta=\gamma$. For $d_{-}=a$, we have $\left(z_{0}, z_{1}\right)=( \pm 1, \mp s)$ and $\alpha=0, \beta=\gamma$.

b) By Lemma 4.2 , we can also have $\left(z_{0}, z_{1}\right)=( \pm c r \mp s t, \mp 1)$, i.e. $\left(z_{0}, z_{1}\right)=$ $\left( \pm w_{-}, \mp 1\right)$. By $(2.7)$, we get the bound for $\gamma$. Specially, for $d_{-}=0$, we get $z_{0}= \pm 1$ and $\alpha \leq \beta=\gamma$. For $d_{-}=a$, we have ${ }^{6}\left(z_{0}, z_{1}\right)=( \pm s, \mp 1), \alpha=0$ and $\beta=\gamma$.

c) By Lemma 4.2, we further have $s z_{0} \pm c x_{0}=t z_{1} \pm c y_{1}$, where $x_{0}$ and $y_{1}$ are not constant and polynomials on both sides of the equation have degree less than $\gamma$. Similarly as for the cases 2.) and 3.), we conclude that $s z_{0}-c x_{0}=$ $t z_{1}-c y_{1}$, where $z_{0}>0$ and $z_{1}>0$, or $s z_{0}+c x_{0}=t z_{1}+c y_{1}$, where $z_{0}<0$ and $z_{1}<0$. Here we have $v_{1}=w_{1}$. By Lemma $3.4 \mathrm{~d}$ ), this can lead to $d_{-}$ or to $d_{+}$if $\gamma \geq \alpha+2 \beta$ or to an irregular $D(1)$-quadruple $\{a, b, c, d\}$, where $d=0 \neq d_{-}$or $d=a \neq d_{-}$and $a \in \mathbb{R}^{+}$. Cases where we obtain $d_{-}$are described in part 3.d) of Lemma 3.5. By (2.7), we obtain $\gamma \geq \alpha+2 \beta$. Cases where we obtain $d_{+}$are possible only if equations $s z_{0} \pm c x_{0}=t z_{1} \pm c y_{1}$ hold for both signs \pm , as it is described in the proof of [18, Proposition 1]. Then, $1])$.

${ }^{6} \operatorname{In} \mathbb{C}[X]$ here appears an irregular polynomial $D(1)$-quadruple $\mathcal{D}_{p}$ (see $[18$, Proposition 
we have $\left(z_{0}, z_{1}\right)=(t, s)$. If $d=0 \neq d_{-}$or $d=a \neq d_{-}$and $a$ is a constant, then $s z_{0} \pm c x_{0}= \pm 1$ or $s z_{0} \pm c x_{0}= \pm s$, respectively. Both cases are not possible, as we have seen in 2.).

\section{Proof of TheOREM 1.4}

We want to find all extensions of an arbitrary $D(1)$-triple $\{a, b, c\}$, with $a<b<c$, in $\mathbb{R}[X]$ to a $D(1)$-quadruple $\{a, b, c, d\}$ in $\mathbb{R}[X]$. By Lemma 2.1, we reduced the problem of finding these extensions to the problem of existence of a suitable solution of equation $(2.15)$, where $\left(v_{m}\right)_{m>0}$ and $\left(w_{n}\right)_{n>0}$ are binary recurrence sequences defined by (2.13) and (2.14), for some initial values $\left(z_{0}, x_{0}\right)$ and $\left(z_{1}, y_{1}\right)$. In Lemma 4.3 we have described all possible initial terms. We will prove that neither of them leads to an irregular $D(1)$ quadruple with $d=d^{\prime}$. As previously stated, $\left\{a, b, c, d^{\prime}\right\}$ is irregular quadruple (where $a<b<c$, with $\operatorname{deg}\left(d^{\prime}\right)=\delta$ and $\gamma \leq \delta$ ) with minimal possible $\delta$ among all irregular $D(1)$-quadruples in $\mathbb{R}[X]$. Recall once again that $r, s, t \in \mathbb{R}^{+}[X]$ are polynomials satisfying $a b+1=r^{2}, a c+1=s^{2}$ and $b c+1=t^{2}$.

Relations from the following lemma are obtained by considering the sequences $\left(v_{m}\right)_{m>0}$ and $\left(w_{n}\right)_{n>0}$ modulo $4 c^{2}$ (see [13, Lemma 6$]$ ). We consider congruences in $\mathbb{R}[X]$.

Lemma 5.1. Let the sequences $\left(v_{m}\right)_{m \geq 0}$ and $\left(w_{n}\right)_{n \geq 0}$ be given by (2.13) and (2.14). Then,

$$
\begin{aligned}
v_{2 m} & \equiv z_{0}+2 c\left(a z_{0} m^{2}+s x_{0} m\right)\left(\bmod c^{2}\right), \\
v_{2 m+1} & \equiv s z_{0}+c\left[2 a s z_{0} m(m+1)+x_{0}(2 m+1)\right]\left(\bmod c^{2}\right), \\
w_{2 n} & \equiv z_{1}+2 c\left(b z_{1} n^{2}+t y_{1} n\right)\left(\bmod c^{2}\right), \\
w_{2 n+1} & \equiv t z_{1}+c\left[2 b t z_{1} n(n+1)+y_{1}(2 n+1)\right]\left(\bmod c^{2}\right) .
\end{aligned}
$$

We will also use the following result, which follows directly from (3.1).

Lemma 5.2. Let $\{a, b, c\}$, with $a<b<c$, be (possibly improper) $D(1)$ triple from $\mathbb{R}[X]$ for which (2.1) holds. Let $d_{-}$be defined by (3.1). Then

$$
2 r s t \equiv a+b-d_{-}(\bmod c) .
$$

Proof of Theorem 1.4. CASe 1.A) $v_{2 m}=w_{2 n}, z_{0}=z_{1}= \pm 1$.

By (2.11), (2.12) and Remark 2.2, we have $x_{0}=1$ and $y_{1}=1$. By Lemma 5.1,

$$
\pm a m^{2}+s m \equiv \pm b n^{2}+t n(\bmod c) .
$$

For $0 \in\{m, n\}$ we obtain an improper $D(1)$-quadruple $\{0, a, b, c\}$, which can be regular or irregular. Hence, we assume that $m, n \neq 0$. Similarly as in [13], by (2.16) and (2.18), we have $\operatorname{deg}\left(v_{2 m}\right)=\gamma+(2 m-1) \frac{\alpha+\gamma}{2}, \operatorname{deg}\left(w_{2 n}\right)=$ $\gamma+(2 n-1) \frac{\beta+\gamma}{2}$, except for $\alpha \leq \beta=\gamma, z_{0}=-1$ and $c=a+b+2 r$, where $\operatorname{deg}\left(v_{2 m}\right)=\gamma+(2 m-1) \frac{\alpha+\gamma}{2}, \operatorname{deg}\left(w_{2 n}\right)=\frac{\alpha+\beta}{2}+(2 n-1) \frac{\beta+\gamma}{2}$. We also have 
to consider the case where $c=b+2 r s$ and $\alpha=0$, which does not exist in [13]. We distinguish subcases $\beta<\gamma$ and $\beta=\gamma$. For $\beta<\gamma$, we obtain a contradiction analogously as in [13].

For $\alpha<\beta=\gamma$, by Lemma 3.5, we have $d_{-}=0$ or $d_{-}=a$ and $\alpha=0$. For $d_{-}=0$ we obtain $d=d_{+}=4 r(a+r)(b+r)$ completely analogously as in [13]. For $d_{-}=a$, using (5.2), (3.18), (3.17) and (2.1), we obtain $\pm a m^{2}+s m \equiv$ $\mp 2 p n^{2}-n(\bmod c)$. Hence, $\pm a m^{2}+s m=\mp 2 p n^{2}-n$, which is not possible, because on the right hand side we have a constant and on the left hand side a non-constant polynomial.

For $\alpha=\beta=\gamma$, completely analogously as in [13], we obtain an improper $D(1)$-quadruple $\{0, a, b, c\}$, which can be regular or irregular or we obtain $d=d_{+}$.

CASE 1.B) $v_{2 m}=w_{2 n}, z_{0}=z_{1}= \pm s$ and $\alpha=0$.

By $(2.11),(2.12)$ and Remark 2.2 , we have $x_{0}^{2}=a^{2}+1$ and $y_{1}=r$. From Lemma 5.1, we have

$$
\pm a s m^{2}+s x_{0} m \equiv \pm b s n^{2}+\operatorname{trn}(\bmod c) .
$$

For $0 \in\{m, n\}$ we obtain an improper $D(1)$-quadruple $\{a, a, b, c\}$, which can be regular or irregular. Hence, we assume that $m, n \neq 0$. By multiplying the congruence (5.3) by $s$ and using (2.1) and (5.1) yields

$$
\pm a m^{2}+x_{0} m \equiv \pm b n^{2}+\frac{a n}{2}+\frac{b n}{2}-\frac{d_{-} n}{2}(\bmod c) \text {. }
$$

Let $\beta<\gamma$. By Lemma 3.3, (5.4) implies

$$
d_{-}=a+b \pm 2 b n \mp 2 \frac{a m^{2}}{n}-2 \frac{x_{0} m}{n} .
$$

If $\operatorname{deg}\left(d_{-}\right)<\beta$, then $1 \pm 2 n=0$, which is not possible. Hence, $\operatorname{deg}\left(d_{-}\right)=\beta$ and by Lemma 3.3, we get $\gamma=2 \beta$. Lemma 3.7 implies $d_{-}=a+b \pm 2 r$ or $d_{-}=b \frac{D_{-}}{B} \pm 2 \frac{\sqrt{D_{-}}}{\sqrt{B}}$. For $d_{-}=a+b \pm 2 r$, by (5.5), $\pm b n \mp r$ is a constant, which is not possible. For $d_{-}=b \frac{D_{-}}{B} \pm 2 \frac{\sqrt{D_{-}}}{\sqrt{B}}$, by $(5.5)$, we conclude that $\frac{D_{-}}{B}=1 \pm 2 n$. Hence,

$$
\frac{D_{-}}{B}=1+2 n
$$

and $z_{0}=z_{1}=s$. By (2.13) and (2.14), $v_{1}=s^{2}+c x_{0}$ and $w_{1}=s t+c r$, so $\operatorname{deg}\left(v_{1}\right)=2 \beta$ and $\operatorname{deg}\left(w_{1}\right)=\frac{5 \beta}{2}$. By (2.16) and (2.18), we furthermore have $\operatorname{deg}\left(v_{2 m}\right)=2 \beta+(2 m-1) \beta$ and $\operatorname{deg}\left(w_{2 n}\right)=\frac{5 \beta}{2}+(2 n-1) \frac{3 \beta}{2}$. From $\operatorname{deg}\left(v_{2 m}\right)=\operatorname{deg}\left(w_{2 n}\right)$, we get $2 m=3 n$. By inserting that into (5.5), we further conclude that $a-\frac{9 a n}{2}-3 x_{0}= \pm 2 \frac{\sqrt{D_{-}}}{\sqrt{B}}$. Since the left hand side of this equation is $<0$, we conclude that $d_{-}=b \frac{D_{-}}{B}-2 \frac{\sqrt{D_{-}}}{\sqrt{B}}$. In that case $B>D_{-}$, thus by $(5.6), 2 n<0$, which is not possible. 
Let $\beta=\gamma$. By Lemma 3.5, $c=a+b+2 r$ and $d_{-}=0$ or $c=b+2 r s$ and $d_{-}=a$. Let $c=a+b+2 r$. From (5.4), we obtain $\pm a m^{2}+x_{0} m \mp b n^{2}-\frac{a n}{2}-\frac{b n}{2}=$ $k(a+b+2 r)$, where $k \in \mathbb{R}$. Hence, $\mp b n^{2}-\frac{b n}{2}-k b-2 k r$ is a constant. From that, by observing degrees, we get $k=0$ and $\mp n^{2}-\frac{n}{2}=0$, which is not possible. Let $c=b+2$ rs. By (3.15), $c=\frac{b}{p^{2}}+\frac{2}{p}$. By (5.4), we get $\pm a m^{2}+x_{0} m \equiv \pm b n^{2}+\frac{b n}{2}(\bmod c)$, so $\pm a m^{2}+x_{0} m \mp b n^{2}-\frac{b n}{2}=k\left(\frac{b}{p^{2}}+\frac{2}{p}\right)$, where $k \in \mathbb{R}$. From that, by comparing degrees of polynomials, we get

$$
\begin{aligned}
& \mp n^{2}-\frac{n}{2}-\frac{k}{p^{2}}=0, \\
& \pm a m^{2}+x_{0} m-\frac{2 k}{p}=0 .
\end{aligned}
$$

If we have the upper combination of signs in (5.7), then from the first equation we get $k<0$ and from the second equation we get $k>0$, which is a contradiction. For the lower combination of signs, $z_{0}=z_{1}=-s$ and $k>0$. By (2.13) and (2.14), we have $v_{1}=\left(x_{0}-a\right) c-1$ and $w_{1}=c r-s t$, so $\operatorname{deg}\left(v_{1}\right)=\gamma$ and by (4.5), $\operatorname{deg}\left(w_{1}\right)=\frac{\gamma}{2}$. From (2.16) and (2.18), we get $\operatorname{deg}\left(v_{2 m}\right)=\gamma+(2 m-1) \frac{\gamma}{2}$ and $\operatorname{deg}\left(w_{2 n}\right)=\frac{\gamma}{2}+(2 n-1) \gamma$. Moreover, $\operatorname{deg}\left(v_{2 m}\right)=\operatorname{deg}\left(w_{2 n}\right)$ implies $m=2 n-1$. Multiplying the first equation in (5.7) by $-2 p$ and then by adding those equations, we obtain $m=\frac{x_{0}-n p}{a}$. Since $p=x_{0}-a$, we have $n-1=\frac{x_{0}}{a}(1-n)$. For $n>1$, we get $x_{0} \stackrel{a}{=}-a$, which is not possible. For $n=1$, we have $m=1$, thus $z=v_{2}=w_{2}$. Similarly as in [18, Proposition 1] we obtain $d=d_{+}$.

CASE 1.C) $v_{2 m}=w_{2 n}, z_{0}=z_{1}= \pm c r \mp s t$ and $\alpha>0, \alpha+\beta \leq \gamma \leq 2 \alpha+\beta$.

From (2.11), (2.12) and Remark 2.2, we have $x_{0}=r s-a t$ and $y_{1}=r t-b s$. For $\beta=\gamma$, we have $\alpha \leq 0$ which is not possible. For $\beta<\gamma$, similarly as in [13], we obtain $d=d_{-}$and $d=d_{+}$.

CASE 2.A $) v_{2 m+1}=w_{2 n},\left(z_{0}, z_{1}\right)=( \pm 1, \pm s)$ and $\gamma \geq 2 \alpha+\beta$.

By (2.11), (2.12) and Remark 2.2, we get $x_{0}=1, y_{1}=r$ and $z_{0} z_{1}>0$. By Lemma 5.1, (5.1) and (1.1), we conclude

$$
\pm 2 a m(m+1)+s(2 m+1) \equiv \pm 2 b n^{2}+a n+b n-d_{-} n(\bmod c) .
$$

Let $\beta<\gamma$. Lemma 3.5 implies $d_{-} \neq 0$ and, by Lemma 3.3, $\operatorname{deg}\left(d_{-}\right) \geq \alpha$. For $n=0$, from (5.8), we get $m=-\frac{1}{2}$ which is not possible, so $n>0$. By (5.8), we obtain

$$
d_{-}= \pm 2 b n+a+b \mp 2 a \frac{m(m+1)}{n}-s \frac{2 m+1}{n} .
$$

For $\beta<\frac{\alpha+\gamma}{2}$, from (5.9), we get $D_{-}=-\sqrt{A C} \frac{2 m+1}{n}<0$, which is not possible. Hence, $\beta \geq \frac{\alpha+\gamma}{2}$. If $\beta>\frac{\alpha+\gamma}{2}$, then by (5.9), $\operatorname{deg}\left(d_{-}\right)=\beta$. By Lemma 3.3, we get $\gamma=\alpha+2 \beta$, which is not possible. Therefore, $\beta=\frac{\alpha+\gamma}{2}$, i.e.

$$
\gamma=2 \beta-\alpha
$$


and $\operatorname{deg}\left(d_{-}\right)=\beta-2 \alpha \leq \beta$. Since $\gamma \geq 2 \alpha+\beta$, we also have

$$
3 \alpha \leq \beta \text {. }
$$

For $\alpha=0$, we have $\gamma=2 \beta$ and $\operatorname{deg}\left(d_{-}\right)=\beta$. By (5.9), we conclude

$$
D_{-}=B( \pm 2 n+1)-\sqrt{A C} \frac{2 m+1}{n} \text {. }
$$

Hence, $z_{0}=1$ and $z_{1}=s$. For $\alpha>0, \operatorname{deg}\left(d_{-}\right)<\beta$, thus $B( \pm 2 n+1)=$ $\sqrt{A C} \frac{2 m+1}{n}$. Again, we must have $z_{0}=1$ and $z_{1}=s$. By (2.13) and (2.14), $v_{1}=s+c$ and $w_{1}=s t+c r$, so $\operatorname{deg}\left(v_{1}\right)=\gamma$ and $\operatorname{deg}\left(w_{1}\right)=\gamma+\frac{\alpha+\beta}{2}$. By (2.16) and (2.18), we get

$$
\operatorname{deg}\left(v_{2 m+1}\right)=\gamma+2 m \beta
$$

and

$$
\operatorname{deg}\left(w_{2 n}\right)=\beta+n(\beta+\gamma) .
$$

From (5.10), (5.13) and (5.14), we have

$$
\alpha(-1+n)=\beta(-1+3 n-2 m) .
$$

Let $\alpha=0$ and $\gamma=2 \beta$. From (5.15), we get

$$
2 m-3 n=-1 \text {. }
$$

Moreover, we have one of the cases from Lemma 3.7. If $d_{-}=a+b \pm 2 r$, then $s=2 r^{2} \pm 2 a r-1$. Hence, $D_{-}=B$ and $C=4 A B^{2}$. From (5.12), using (5.16), we obtain $n=3 a$. Furthermore, from (5.9), we get $r( \pm 2 \pm 6 a)=$ $-\frac{2}{3} m(m+1)-3$. Hence, $-\frac{2}{3} m(m+1)=3$, which is not possible. Let $d_{-}=b \frac{D_{-}}{B} \pm 2 \frac{\sqrt{D_{-}}}{\sqrt{B}}$. By comparing the leading coefficients in (5.9), using (5.16) and the equation

$$
C=4 A B D_{-},
$$

obtained by comparing the leading coefficients in (3.4), we obtain

$$
\left(D_{-}-B\right)(1 \pm 3)=2 B n \text {. }
$$

If in (5.18) we have the sign - , then $d_{-}<b$ and $-D_{-}=B(n-1)$. Since $D_{-}>0$, we get $n<1$, which is not possible. If in (5.18) we have the sign + , then $d_{-}>b$ and $\frac{D_{-}}{B}=\frac{n}{2}+1$. Using that, (3.25) and (5.16), from (5.9), we obtain $\left(D_{-}-B\right)\left(-\frac{m(m+1)}{n}-4\right)=2 D_{-}$. It yields $-\frac{m(m+1)}{n}-4>0$, a contradiction.

Let $\alpha>0$. From Lemma 5.1, we obtain

$$
2 s\left(a m(m+1)-b n^{2}\right) \equiv 2 t r n-(2 m+1)(\bmod c) .
$$

By squaring that, using (1.1), we get

(5.19) $4\left(a m(m+1)-b n^{2}\right)^{2} \equiv 4 r^{2} n^{2}-4 \operatorname{trn}(2 m+1)+(2 m+1)^{2}(\bmod c)$. 
Using (5.9), we define

$$
g:=(2 n+1) b-s \frac{2 m+1}{n}=d_{-}-a\left(1-2 \frac{m(m+1)}{n}\right) .
$$

By observing degrees of polynomials in (5.20), we conclude that $\operatorname{deg}(g) \leq$ $\beta-2 \alpha$. If $\operatorname{deg}(g)<\beta-2 \alpha$, then $\beta=3 \alpha$ and $D_{-}=A\left(1-2 \frac{m(m+1)}{n}\right)$. Hence, $1-2 \frac{m(m+1)}{n}>0$. By (5.15), $\alpha=\beta\left(3+2 \frac{1-m}{n-1}\right)<\beta$. Hence, $n<m$ and we have $1>2(n+1)$, a contradiction. Therefore, $\operatorname{deg}(g)=\beta-2 \alpha$. By (5.20),

$$
b=s \frac{2 m+1}{n(2 n+1)}+\frac{g}{2 n+1} .
$$

Using (3.3) and (5.21), we get

$$
r t=s^{2} \frac{2 m+1}{n(2 n+1)}+\frac{g s}{2 n+1}-v_{-} .
$$

Using (5.21) and (5.22), from (5.19), we obtain

$$
\begin{aligned}
4 a^{2} m^{2}(m+1)^{2}-8 a m(m+1) n^{2}\left(s \frac{2 m+1}{n(2 n+1)}+\frac{g}{2 n+1}\right)+ \\
+4 n^{4}\left(\frac{(2 m+1)^{2}}{n^{2}(2 n+1)^{2}}+2 s g \frac{2 m+1}{n(2 n+1)^{2}}+\frac{g^{2}}{(2 n+1)^{2}}\right) \\
\equiv 4 r^{2} n^{2}-4 n(2 m+1)\left(\frac{2 m+1}{n(2 n+1)}+\frac{g s}{2 n+1}-v_{-}\right) \\
\quad+(2 m+1)^{2}(\bmod c) .
\end{aligned}
$$

By considering degrees of polynomials on both sides of the congruence (5.23), using (5.10) and (5.11), we conclude that (5.23) is an equation. If $\beta>3 \alpha$, then, by considering the leading coefficients in that equation, we get $2 n^{2}=$ $-2 n-1$, which is a contradiction. Let $\beta=3 \alpha$. Using (5.20), from the equation obtained from (5.23), we get that $b\left(-8 a m(m+1) n^{2}+8 \frac{n^{4}}{2 n+1} g-4 a n^{2}+4 n^{2} g\right)$ is a polynomial of degree $<\beta$. This is possible only if $a(2 m(m+1)+1)=g(1+$ $\left.\frac{2 n^{2}}{2 n+1}\right)$. Since $2 m(m+1) \neq-1$, it follows that $a \mid g$. Hence, by $(5.20), d_{-}=\xi a$, where $\xi \in \mathbb{R}$. By $(3.2)$, we have $\xi a^{2}+1=u_{-}^{2}$. Hence, $\left(a-\frac{u_{-}}{\sqrt{\xi}}\right)\left(a+\frac{u_{-}}{\sqrt{\xi}}\right)=-\frac{1}{\xi}$, which is not possible since both factors on the left hand side of this equation are non-constant.

If $\beta=\gamma$, then $\alpha=0$. By Lemma 3.5, $d_{-}=0$ or $d_{-}=a$. If $d_{-}=0$, then, by $(5.8), \pm 2 a m(m+1)+(a+r)(2 m+1) \mp 2 b n^{2}-a n-b n=k(a+b+2 r)$, where $k \in \mathbb{R}$. By comparing degrees on both sides of this equation, we conclude that $\mp 2 n^{2}-n=k$, i.e. $k$ is an integer. Furthermore, $2 m+1=2 k$, which is not possible. If $d_{-}=a$, by (5.8), we have $\pm 2 a m(m+1)+s(2 m+1) \mp 2 b n^{2}-b n=$ $k\left(b+\frac{2 a b}{p}+\frac{2}{p}\right)$, where $k \in \mathbb{R}$. By comparing degrees on both sides of this equation, we conclude that $\mp 2 n^{2}-n=k+\frac{2 k a}{p}$. Further, $2 m+1=0$, which is not possible. 
CASE 2.B) $v_{2 m+1}=w_{2 n}, z_{0}= \pm s, z_{1}= \pm 1$ and $\alpha=0$.

By (2.11), (2.12) and Remark 2.2, we have $x_{0}^{2}=a^{2}+1, y_{1}=1$ and $z_{0} z_{1}>0$. By Lemma 5.1 and (2.1),

$$
\pm a \pm 2 a m(m+1)+\sqrt{a^{2}+1}(2 m+1) \equiv 2\left( \pm b n^{2}+t n\right)(\bmod c) .
$$

If $\beta<\gamma$, the congruence (5.24) is actually an equation. The left hand side of that equation is constant, so $n=0$. Hence, $z=w_{0}=z_{1}= \pm 1$, so we obtain an irregular polynomial $D(1)$-quadruple $\{0, a, b, c\}$.

Let $\beta=\gamma$. By Lemma 3.5, $d_{-}=0$ or $d_{-}=a$. If $d_{-}=0$, then, by (5.24), (1.3) and (1.4), we have $\pm a \pm 2 a m(m+1)+\sqrt{a^{2}+1}(2 m+1)-2\left( \pm b n^{2}+\right.$ $(b+r) n)=k(a+b+2 r)$, where $k \in \mathbb{R}$. By comparing the leading coefficients on both sides of this equation, we obtain $-n=k$ and $\mp 2 n^{2}-2 n=k$. Therefore, $n=0$ or $\mp 2 n-1=0$, which is not possible. For $n=0$ we get a regular polynomial $D(1)$-quadruple $\{0, a, b, c\}$. If $d_{-}=a$, then, by (5.24) and Remark 3.6, we get $\pm a \pm 2 a m(m+1)+\sqrt{a^{2}+1}(2 m+1)-2( \pm$ $\left.b n^{2}+\left(\frac{b}{p}+1\right) n\right)=k\left(\frac{b}{p^{2}}+\frac{2}{p}\right)$, where $k \in \mathbb{R}$. By comparing the leading coefficients on both sides of this equation, we obtain $\mp 2 n^{2}-\frac{2 n}{p}=\frac{k}{p^{2}}$ and $\pm a \pm 2 a m^{2} \pm 2 a m+2 m x_{0}+x_{0}-2 n=\frac{2 k}{p}$. From that we have

$$
\left(x_{0} \pm a\right)(1+2 m) \pm 2 a m^{2}=\mp 4 n^{2} p-2 n .
$$

Since $x_{0}=-u_{-}$and $0<p<1$, for $z_{0}=s$ and $z_{1}=1$, from (5.25) we get $\frac{1}{p}(1+2 m)+2 a m^{2}=-4 n^{2} p-2 n$, where the left hand side is $>1$ and the right hand side is $\leq 0$, which is not possible. Hence, $z_{0}=-s$ and $z_{1}=-1$. In that case, from (5.25), we get $p\left(1+2 m-4 n^{2}\right)=2 a m^{2}-2 n$ and then

$$
1>2 m(a m-1)+2 n(2 n-1) .
$$

By (2.13) and (2.14), we have $v_{1}=c p-1$ and $w_{1}=-t+c, \operatorname{so} \operatorname{deg}\left(v_{1}\right)=$ $\operatorname{deg}\left(w_{1}\right)=\gamma$. By $(2.16),(2.18)$ and $\operatorname{deg}\left(v_{2 m+1}\right)=\operatorname{deg}\left(w_{2 n}\right)$, we obtain

$$
m+1=2 n \text {. }
$$

From (5.26), we get $1>m^{2}(2 a+1)-m$. Since $a>0$, we have $1>m(m-1)$, which is not possible for $m \geq 2$. For $m=0$, by (5.27), $n=\frac{1}{2}$ which is also not possible. For $m=1$, by (5.27), we get $n=1$ and we have $v_{3}=w_{2}$. By [18], this case is not possible.

CASE 2.C) $v_{2 m+1}=w_{2 n}, z_{0}=-t, z_{1}=c r-s t$ and $\alpha=\beta, \gamma=3 \alpha$.

By (2.11), (2.12) and Remark 2.2, we have $x_{0}=r, y_{1}=-v_{-}=r t-b s$. Since $v_{1}=-s t+c r$, we have $\operatorname{deg}\left(v_{1}\right)=\gamma-\frac{\alpha+\beta}{2}$. Similarly as in [13, Lemma $8], \operatorname{deg}\left(w_{1}\right)=\frac{3 \gamma-\alpha}{2}$. By $(2.16),(2.18)$ and $\operatorname{deg}\left(v_{2 m+1}\right)=\operatorname{deg}\left(w_{2 n}\right)$, we obtain

$$
m=n \text {. }
$$

By Lemma 5.1 and (5.28), we conclude $-2 a s t m(m+1) \equiv-2 b s t\left(m^{2}+\right.$ $m)(\bmod c)$. By multiplying this congruence by $s t$, we get $-2 a m(m+1) \equiv$ $-2 b\left(m^{2}+m\right)(\bmod c)$. Since $\beta<\gamma$, from that we obtain $2 m(m+1)(-a+b)=0$. 
For $m=0$, we have $z=z_{1}=w_{-}$, so $d=d_{-}$. The cases $m=-1$ and $a=b$ are not possible.

CASE 3.A) $v_{2 m}=w_{2 n+1}, z_{0}= \pm t, z_{1}= \pm 1$ and $\gamma \geq \alpha+2 \beta$.

By (2.11), (2.12) and Remark 2.2, we have $x_{0}=r, y_{1}=1$ and $z_{0} z_{1}>0$. By Lemma 5.1, (5.1) and (1.1), we conclude

$$
\pm 2 a m^{2}+a m+b m-d_{-} m \equiv \pm 2 b n(n+1)+t(2 n+1)(\bmod c) .
$$

In this case $\beta \leq \operatorname{deg}\left(d_{-}\right)<\gamma$. Hence, (5.29) becomes an equation. If $\operatorname{deg}\left(d_{-}\right)<\frac{\beta+\gamma}{2}$, then, by considering leading coefficients of polynomials on both sides of the equation, we get $2 n+1=0$, a contradiction. If $\operatorname{deg}\left(d_{-}\right)>\frac{\beta+\gamma}{2}$, then $m=0$, so $z=v_{0}=z_{0}= \pm t$. Hence, $d=b$, which is not possible. Therefore, $\operatorname{deg}\left(d_{-}\right)=\frac{\beta+\gamma}{2}$. By considering the leading coefficients in equation obtained from (5.29), we get $-D_{-} m=\sqrt{B C}(2 n+1)$. This is not possible, since on the left hand side of this equation we have a real number $\leq 0$ and the right hand side is $>0$.

CASE 3.B) $v_{2 m}=w_{2 n+1}, z_{0}= \pm s, z_{1}=\mp 1$ and $\alpha=0, \beta=\gamma$.

By (2.11), (2.12) and Remark 2.2, we have $x_{0}^{2}=a^{2}+1, y_{1}=1$ and $z_{0} z_{1}<0$. From the proof of Lemma 4.3, we know that $d_{-}=0$, so $c=a+b+2 r$. Using that and (1.4), by Lemma 5.1, we obtain

$$
\pm 1+2 s\left( \pm a m^{2}+x_{0} m\right) \equiv \mp 2 b t n(n+1)+2 n+1(\bmod c) .
$$

Since, by $(1.4)$, in this case $s t \equiv-1(\bmod c)$, by multiplying $(5.30)$ by $t$, we obtain

$(5.31) \pm(b+r) \mp 2 a m^{2}-2 x_{0} m \pm 2 b n(n+1)-(2 n+1)(b+r)=k(a+b+2 r)$,

where $k \in \mathbb{R}$. By comparing degrees of polynomials on both sides of the equation (5.31), we get

$$
\begin{aligned}
\pm 1 \pm 2 n(n+1)-(2 n+1) & =k, \\
\pm 1-(2 n+1) & =2 k, \\
\mp 2 a m^{2}-2 x_{0} m & =k a .
\end{aligned}
$$

From the first two equations in (5.32), we get $\pm 1-2 n-1=\mp 4 n(n+1)$, where only the upper combination of signs is possible, i.e. we have $-n=2 n^{2}$. Hence, $n=0$ and $k=0$. Using that, from the third equation in (5.32), we get $a m^{2}+x_{0} m=0$. Since $a m+x_{0}>0$, we have $m=0$. Therefore, $z=v_{0}=z_{0}= \pm s$ and we obtain $d=a$, i.e. $\{a, a, b, c\}$ is an irregular polynomial $D(1)$-quadruple.

CASE 3.C) $v_{2 m}=w_{2 n+1}, z_{0}= \pm 1, z_{1}= \pm 1$, with $z_{0} z_{1}>0$, and $\alpha=0$, $\beta=\gamma$.

By (2.11), (2.12) and Remark 2.2, $x_{0}=1$ and $y_{1}=1$. By $(2.13), v_{1}=$ $\pm s+c$, so $\operatorname{deg}\left(v_{1}\right)=\gamma$. By the proof of Lemma 4.2, $d_{-}=a$. By (2.14), using (3.20), we get $w_{1}= \pm t+c=c( \pm p+1) \mp 1$. Hence, $\operatorname{deg}\left(w_{1}\right)=\gamma$. 
By (2.16) and (2.18), $\operatorname{deg}\left(v_{2 m}\right)=m \gamma+\frac{\gamma}{2}$ and $\operatorname{deg}\left(w_{2 n+1}\right)=2 n \gamma+\gamma$. From $\operatorname{deg}\left(v_{2 m}\right)=\operatorname{deg}\left(w_{2 n+1}\right)$, we get $m-2 n=\frac{1}{2}$, which is not possible.

CASE 3.D) $v_{2 m}=w_{2 n+1}, z_{0}=c r-s t, z_{1}=-s$ and $\alpha \geq 0,2 \alpha+\beta \leq \gamma \leq$ $\alpha+2 \beta$.

By (2.11), (2.12) and Remark 2.2, we get $x_{0}=-u=r s-a t, y_{1}=r$. Similarly as in [13, Lemma 8], $\operatorname{deg}\left(v_{1}\right)=\frac{3 \gamma-\beta}{2}$, so $\operatorname{deg}\left(v_{2 m}\right)=\frac{3 \gamma-\beta}{2}+(2 m-$ 1) $\frac{\alpha+\gamma}{2}$. By (2.14), $w_{1}=c r-s t, \operatorname{deg}\left(w_{1}\right)=\gamma-\frac{\alpha+\beta}{2}$ and $\operatorname{deg}\left(w_{2 n+1}\right)=$ $\gamma-\frac{\alpha+\beta}{2}+n(\beta+\gamma)$. From $\operatorname{deg}\left(v_{2 m}\right)=\operatorname{deg}\left(w_{2 n+1}\right)$, we get

$$
m(\alpha+\gamma)=n(\beta+\gamma) \text {. }
$$

By Lemma 5.1, similarly as in previous cases, we obtain

$$
-2(\operatorname{am}(m+1)-b n(n+1)) \equiv 2 r s t(n-m)(\bmod c) .
$$

Assume firstly that $\gamma=\alpha+2 \beta$. By Lemma 3.7, the triple $\left\{a, b, d_{-}\right\}$has the form 1.) or 2.a) from Lemma 3.5. Let $d_{-}=a+b \pm 2 r$. By (5.1), from (5.34), we get

$$
-2(a m(m \pm 1)-b n(n+1))= \pm 2 r(n-m) .
$$

If $\alpha<\beta$, from (5.35), we get $n(n+1)=0$. The case $n=-1$ is not possible and for $n=0$, by (5.33), we get $m=0$. For $v_{0}=w_{1}$, we have $z=z_{0}=c r-s t$ and $d=d_{-}$. If $\alpha=\beta$, then $\gamma=3 \alpha$. From (5.33) and (5.35), we have $m=n$ and then $m(m+1)(-2 a+2 b)=0$. Since $m \geq 0$ and $a \neq b$, we get $m=n=0$. Hence, again $z=v_{0}=w_{1}$. If the triple $\left\{a, b, d_{-}\right\}$has the form 2.a) from Lemma 3.5, then $\alpha=0, \gamma=2 \beta$. By Lemma 3.7, we have $d_{-}=b \pm 2 r^{2} \frac{\sqrt{D_{-}}}{\sqrt{B}}$. Using (5.1) and (1.1), from (5.34), we get

$$
-2(a m(m \pm 1)-b n(n+1))=\left(a \mp 2(a b+1) \frac{\sqrt{D_{-}}}{\sqrt{B}}\right)(n-m) .
$$

By comparing degrees of polynomials on both sides of the equation (5.36), we obtain $a(-2 m(m \pm 1)-n+m)=\mp 2 \frac{\sqrt{D_{-}}}{\sqrt{B}}(n-m)$ and $2 n(n+1)=$ $\mp 2 a \frac{\sqrt{D_{-}}}{\sqrt{B}}(n-m)$. From that, it follows

$$
a^{2}(-2 m(m \pm 1)-n+m) \mp 2 n(n+1)=0 .
$$

From (5.33) and (5.37), we get $2 m=3 n$ and then $m=n=0$ (which we already had) or $n>0$ and $a^{2}=\frac{4(n+1)}{-9 n-5}$, a contradiction.

Let $\gamma<\alpha+2 \beta$. Then $\alpha<\beta$ and, by Lemma 3.3, we have $\operatorname{deg}\left(d_{-}\right)<\beta$. Using (5.1), from (5.34), we get

$$
-2(a m(m \pm 1)-b n(n+1))=\left(a+b-d_{-}\right)(n-m) .
$$

By comparing degrees of polynomials on both sides of the equation (5.38), we obtain $m=n(-2 n-1)$, which is possible only for $m=n=0$. This leads to $d=d_{-}$. For $d_{-}=a$, we have the case 3.d)1). 
CASE 4.A) $v_{2 m+1}=w_{2 n+1}, z_{0}= \pm 1, z_{1}=\mp c r \pm s t$ and $\gamma \leq 2 \alpha+\beta$.

By (2.11), (2.12) and Remark 2.2, $x_{0}=1, y_{1}=r t-b s$ and $z_{0} z_{1}<0$. If $z_{0}>0$ and $z_{1}<0$ then, by (2.13), $v_{1}=s+c \operatorname{so} \operatorname{deg}\left(v_{1}\right)=\gamma$. By $(2.14)$, similarly as in [13, Lemma 8], we get $\operatorname{deg}\left(w_{1}\right)=\frac{\alpha+\gamma}{2}$. If $z_{0}<0$ and $z_{1}>0$ then, by (2.13), we have $v_{1}=-s+c$. Since $a<c, \operatorname{deg}\left(v_{1}\right)=\gamma$. By (2.14), similarly as in [13, Lemma 8], we get $\operatorname{deg}\left(w_{1}\right)=\frac{3 \gamma-\alpha}{2}$. From (2.16), (2.18) and $\operatorname{deg}\left(v_{2 m+1}\right)=\operatorname{deg}\left(w_{2 n+1}\right)$, for $z_{0}>0$ and $z_{1}<0$, we get

$$
m(\alpha+\gamma)=\frac{\alpha-\gamma}{2}+n(\beta+\gamma)
$$

Also, for $z_{0}<0$ and $z_{1}>0$, we get $m(\alpha+\gamma)=\frac{\gamma-\alpha}{2}+n(\beta+\gamma)$. By Lemma 5.1, (5.1) and (1.1), similarly as in the previous cases, we obtain

$$
\begin{aligned}
\pm 2 a m(m+1)+s(2 m+1) \equiv & \left(\frac{a}{2}+\frac{b}{2}-\frac{d_{-}}{2}\right)\left(2 n+\left\{\begin{array}{l}
0 \\
2
\end{array}\right\}\right) \\
& +b\left( \pm 2 n^{2}+\left\{\begin{array}{c}
0 \\
-4 n-2
\end{array}\right\}\right)(\bmod c) .
\end{aligned}
$$

Let $\beta<\gamma$. We have $\alpha>0$, since otherwise $\gamma \leq \beta$. By Lemma 3.3, we have $0 \leq \operatorname{deg}\left(d_{-}\right) \leq \alpha$. Hence, in (5.40) we have an equation. For $\frac{\alpha+\gamma}{2}>\beta$, by comparing degrees in that equation, we get $2 m+1=0$, which is not possible. Hence, $\frac{\alpha+\gamma}{2} \leq \beta$, so $\alpha<\beta$. For $\frac{\alpha+\gamma}{2}<\beta$, by comparing degrees in equation obtained from (5.40), for $z_{0}>0$ and $z_{1}<0$, we get $n(1+2 n)=0$, i.e. $n=0$. By (5.39), this is not possible. For $z_{0}<0$ and $z_{1}>0$, we get $2 n^{2}+3 n+1=0$, i.e. $n=-\frac{1}{2}$ or $n=-1$, a contradiction in both cases. We are left with the possibility that $\frac{\alpha+\gamma}{2}=\beta$, i.e.

$$
\gamma=2 \beta-\alpha
$$

and $\operatorname{deg}\left(d_{-}\right)=\beta-2 \alpha$. From that, we get

$$
2 \alpha \leq \beta \leq 3 \alpha .
$$

For $z_{0}>0$ and $z_{1}<0$, from (5.40), we obtain the equation (5.9). By comparing degrees in that equation, we obtain

$$
B n(2 n+1)=\sqrt{A C}(2 m+1) .
$$

Since $m$ is a nonnegative integer, we conclude that $n>0$. By (5.39) and (5.41), we get (5.15). From Lemma 5.1, similarly as in the case 2.a), we obtain the congruence (5.19). Again, we use a polynomial $g$ defined by (5.20), by which from (5.19) we get the congruence (5.23). By considering degrees of polynomials which appear in (5.20), similarly as for the case 2.a), we conclude that $\operatorname{deg}(g)=\alpha$. By considering degrees of polynomials which appear in (5.23), we conclude that if $\beta>2 \alpha$, then (5.23) becomes an equation. By considering leading coefficients in that equation, we get a contradiction similarly as in the case 2.a). By (5.42), we are left with the possibility that $\beta=2 \alpha$ and $d_{-}$is a non-zero constant. In this case we can apply Lemma 3.7 
to a $D(1)$-triple $\left\{d_{-}, a, b\right\}$ and we have to observe two possibilities. The first possibility, by Lemma 3.7, is that we have

$$
b=4 u_{-}\left(u_{-} \pm d_{-}\right)\left(a \pm u_{-}\right) .
$$

By considering leading coefficients of polynomials on both sides of the equation (5.44), we conclude that $B=4 A^{2} D_{-}$. Using that and (5.17), from (5.43), we obtain

$$
n(2 n+1)=2 m+1 .
$$

For $\beta=2 \alpha$, from (5.15), we get

$$
5 n-4 m=1 .
$$

All nonnegative solutions $(m, n)$ of the equation (5.46) are given by

$$
m=1+5 t, \quad n=1+4 t,
$$

where $t \in \mathbb{N}_{0}$. By inserting (5.47) into (5.45), we obtain $t(16 t+5)=0$. Hence, $t=0$ i.e. $(m, n)=(1,1)$ or $t=-\frac{5}{16}$, which is not possible. For $(m, n)=(1,1)$ we have $v_{3}=w_{3}$. In this case, by (2.13) and (2.14),

$$
s z_{0}+c\left(4 a s z_{0}+3 x_{0}\right)+4 a c^{2} x_{0}=t z_{1}+c\left(4 b t z_{1}+3 y_{1}\right)+4 b c^{2} y_{1} .
$$

From (5.48), using (1.1), we get $2(b s+r t)=4 a s+4 s^{2}-1$. Therefore,

$$
2(r t-b s) \equiv-1(\bmod s) .
$$

Using (1.1), we conclude that

$$
(b s+r t)(b s-r t)=b^{2}-a b-b c-1 .
$$

From (5.50), we furthermore conclude that $\operatorname{deg}(b s-r t)=\frac{\gamma-\alpha}{2}$. Thus, by (5.49), $2(r t-b s)=-1$ i.e. $b s-r t=v_{-}>0$, which is a contradiction. The second possibility, by Lemma 3.7 , is that we have

$$
d_{-}=\frac{1}{2}\left(\frac{1}{p}-p\right)
$$

and

$$
b=d_{-}+4 u_{-}^{2} p(a p-1)
$$

if $e_{-}<a$, or

$$
b=d_{-}+4 \frac{u_{-}^{2}}{p}\left(\frac{a}{p}+1\right),
$$

if $e_{-}>a$, where $0<p<1$ and $e_{-}$is obtained by (3.1) for the triple $\left\{d_{-}, a, b\right\}$. By considering degrees of polynomials on both sides of the equation (5.52), we get $B=4 A^{2} D_{-} p^{2}$. Using that and (5.17), from (5.43), we obtain

$$
p=\frac{2 m+1}{n(2 n+1)}
$$


Analogously, from (5.53), we get $B=\frac{4 A^{2} D_{-}}{p^{2}}$ and then

$$
p=\frac{n(2 n+1)}{2 m+1} \text {. }
$$

From (3.4), using (3.2) and (3.3), for the triple $\left\{d_{-}, a, b\right\}$ we obtain

$$
c=-a+b+d_{-}-2 s v_{-} .
$$

By considering degrees of polynomials which appear in (5.23), using (5.56), we get

$$
\begin{aligned}
4 a^{2} m^{2}(m+1)^{2}-8 a m(m+1) n^{2}\left(s \frac{2 m+1}{n(2 n+1)}+\frac{g}{2 n+1}\right) \\
\quad+4 n^{4}\left(\frac{(2 m+1)^{2}}{n^{2}(2 n+1)^{2}}+2 s g \frac{2 m+1}{n(2 n+1)^{2}}+\frac{g^{2}}{(2 n+1)^{2}}\right)-4 r^{2} n^{2} \\
\quad+4 n(2 m+1)\left(\frac{2 m+1}{n(2 n+1)}+\frac{g s}{2 n+1}-v_{-}\right)-(2 m+1)^{2} \\
=l\left(-a+b+d_{-}-2 s v_{-}\right),
\end{aligned}
$$

where $l \in \mathbb{R}$. A polynomial $v_{-}$plays for the triple $\left\{d_{-}, a, b\right\}$ the same role as polynomial $s$ does for the triple $\{a, b, c\}$. Therefore, by (3.25) (where $s>0$ and $\left.v_{-}<0\right)$, for (5.52) and (5.54) we have

$$
v_{-}=\left(p^{2}-1\right) a-\frac{1}{2}\left(3 p-\frac{1}{p}\right) .
$$

Analogously, for (5.53) and (5.55), we have

$$
v_{-}=\left(1-\frac{1}{p^{2}}\right) a-\frac{1}{2}\left(3 \frac{1}{p}-p\right) .
$$

From (5.20), we get

$$
g=d_{-}-a\left(1-2 \frac{m(m+1)}{n}\right) .
$$

Also, from (5.20), for (5.52) and (5.54) we have

$$
s=\frac{b}{p}-\frac{g n}{2 m+1},
$$

and for (5.53) and (5.55) we have

$$
s=p b-\frac{g n}{2 m+1} .
$$

By inserting (5.47), (5.54), (5.51), (3.3), (5.52), (5.60), (5.58) and (5.61) into the equation (5.57), we get the expression of the form $c_{3} a^{3}+c_{2} a^{2}+c_{1} a+c_{0}$, where $c_{i}$ are rational expressions with unknowns $l$ and $t$, for $i=0, \ldots, 3$. By solving the system $c_{3}=0, c_{2}=0$ in unknowns $l$ and $t$, the only integer $t$ we obtain is $t=0$. But, for $t=0$ the coefficients $c_{1}$ and $c_{0}$ cannot both be equal to 0 . We conclude the same if we insert (5.47), (5.55), (5.51), (3.3), 
(5.53), (5.60), (5.59) and (5.62) into the equation (5.57). For $z_{0}<0$ and $z_{1}>0$, by comparing degrees in the equation obtained from (5.40), we get $\sqrt{A C}(2 m+1)=B\left(-2 n^{2}-3 n-1\right)<0$, which is not possible.

If $\beta=\gamma$, for $d_{-}=0$ we have $z_{1}=\mp 1, \alpha \leq \beta$, and for $d_{-}=a$ we have $z_{1}=\mp s, \alpha=0$. Let $d_{-}=0$. Then, $y_{1}=1$. By Lemma 5.1, using (1.3), (1.4) and the fact that $c=s+t$, similarly as in previous cases, we obtain

$$
\pm 1 \pm 2 \operatorname{asm}(m+1)+2 m \equiv \pm 2 b \operatorname{sn}(n+1)+2 n(\bmod c) .
$$

Multiplying (5.63) by $s$ and by using (1.1), we get

$$
( \pm 1+2 m-2 n) s \pm 2 a m(m+1) \mp 2 b n(n+1)=k(a+b+2 r),
$$

where $k \in \mathbb{R}$. If $\alpha<\beta$, by comparing degrees in (5.64), we first get $k=$ $\mp 2 n(n+1)$. Further, $\pm 1+2 m-2 n=\mp 4 n(n+1)$. If $n \geq 1$, then $2 \mid( \pm 1)$, which is not possible. For $n=0$, we have $\pm 1+2 m=0$, which is also not possible. Therefore, $\alpha=\beta=\gamma$ and $C=A+B+2 \sqrt{A B}$. By (2.13) and (2.14), we have $\operatorname{deg}\left(v_{1}\right)=\operatorname{deg}\left(w_{1}\right)=\gamma$. By $(2.16),(2.18)$ and $\operatorname{deg}\left(v_{2 m+1}\right)=\operatorname{deg}\left(w_{2 n+1}\right)$, we obtain $m=n$. Using that and the fact that $b-a=c-2 s$, from (5.64), we get

$$
s( \pm 1 \pm 4 n(n+1)) \equiv 0(\bmod c) .
$$

Since $\operatorname{gcd}(c, s)=1$, we have $\pm 1 \pm 4 n(n+1)=0$, which is a contradiction. If $d_{-}=a$, then $d_{1}=a$ and $y_{1}=r$. By Lemma 5.1, (1.1), (3.17), (3.18) and the fact that $r=p s$, where $p \in \mathbb{R}$ and $0<p<1$, similarly as in previous cases, we obtain

$(5.66) \pm 2 a m(m+1)+s(2 m+1) \equiv \mp \frac{1}{p} \pm 2 a \mp 4 n(n+1) p+p(2 n+1)(\bmod c)$.

Since degrees of polynomials on both sides of the congruence (5.66) are less than $\gamma$, we get an equation. By considering degrees of polynomials on both sides of that equation, we get $2 m+1=0$, which is not possible.

CASE 4.B) $v_{2 m+1}=w_{2 n+1}, z_{0}= \pm c r \mp s t, z_{1}=\mp 1$ and $\gamma \leq \alpha+2 \beta$.

By (2.11), (2.12) and Remark 2.2, we have $x_{0}=r s-a t, y_{1}=1$ and $z_{0} z_{1}<0$. By Lemma 5.1, (5.1) and (1.1), we furthermore have (5.67)

$\mp 2 a m(m+1)+\left(-\frac{a}{2}+\frac{b}{2}-\frac{d_{-}}{2}\right)\left(2 m+\left\{\begin{array}{l}2 \\ 0\end{array}\right\}\right) \equiv \mp 2 b n(n+1)+t(2 n+1)(\bmod c)$,

where the upper case is for $z_{0}>0, z_{1}<0$ and the lower is for $z_{0}<0, z_{1}>0$.

If $\beta<\gamma$, from (5.67) we obtain an equation. Since $\operatorname{deg}\left(d_{-}\right) \leq \beta$ and $\operatorname{deg}(t)>\beta$, by comparing degrees in that equation, we get $2 n+1=0$, which is not possible.

Let $\beta=\gamma$. For $d_{-}=0$, we have $z_{0}= \pm 1, z_{1}=\mp 1$ and $\alpha \leq \beta$. By $(2.11)$ and (2.12), we get $x_{0}=1$ and $y_{1}=1$. By Lemma 5.1 and (1.1), using (1.3), 
(1.4) and $s t \equiv-1(\bmod c)$, similarly as in previous cases, we obtain

$$
b( \pm 1+2 m \pm 2 n(n+1)-2 n)+r( \pm 1+2 m-2 n)+a(\mp 2 m(m+1))
$$

$$
=k(a+b+2 r),
$$

where $k \in \mathbb{R}$. If $\alpha<\beta$, by comparing the leading coefficients in (5.68), we first obtain $\pm 1+2 m \pm 2 n(n+1)-2 n=k$. Then, $\pm 1+2 m-2 n=2 k$, so $k=\mp 2 n(n+1)$. Furthermore, we get $k=\mp 2 m(m+1)$. Therefore, $n=m$ or $n=-m-1$. For $m=n$, we get $k= \pm \frac{1}{2}$ and then, $\mp 2 m(m+1)= \pm \frac{1}{2}$. This is not possible. The case where $n=-m-1<0$ is also not possible. Hence, $\alpha=\beta=\gamma$. Since $a<b<c$, from (2.13) and (2.14), we conclude that $\operatorname{deg}\left(v_{1}\right)=\operatorname{deg}\left(w_{1}\right)=\gamma$. By $(2.16),(2.18)$ and $\operatorname{deg}\left(v_{2 m+1}\right)=\operatorname{deg}\left(w_{2 n+1}\right)$, we obtain $m=n$. Using that and the fact that $b-a=c-2 s$, from (5.68), we get (5.65) which is not possible as in the case 4.a). For $d_{-}=a$, we have $z_{0}= \pm s$, $z_{1}=\mp 1, \alpha=0$ and $\beta=\gamma$. By (2.11), (2.12) and Remark 3.6, $x_{0}=a+p$, where $p \in \mathbb{R}$ and $0<p<1$, and $y_{1}=1$. Lemma 5.1 implies

$$
\begin{aligned}
\pm s^{2}+c\left( \pm 2 a s^{2} m(m+1)+(a+p)(2 m+1)\right) \equiv & \mp t+c(\mp 2 b t n(n+1) \\
& +2 n+1)\left(\bmod c^{2}\right) .
\end{aligned}
$$

From (3.18) and (3.20), we have

$$
b t \equiv 2 p(\bmod c) .
$$

From (3.19), we obtain $c\left(\frac{1}{p}-a\right)=t+s^{2}$. Using that, and dividing the obtained congruence by $c$, we get

$$
\begin{gathered}
\pm\left(\frac{1}{p}-a\right) \pm 2 a s^{2} m(m+1)+(a+p)(2 m+1) \\
\equiv \mp 2 b t n(n+1)+2 n+1(\bmod c) .
\end{gathered}
$$

From (3.10), we obtain $\frac{1}{p}-a=p+a$. Using that, (5.69) and (2.1), by (5.70), we conclude

$$
(p+a)( \pm 1+2 m+1) \pm 2 a m(m+1)=\mp 4 p n(n+1)+2 n+1 .
$$

By (2.13), $v_{1}= \pm 1+c( \pm a+a+p)$ and by (2.14) and (3.20), $w_{1}=t(\mp 1+$ $\left.\frac{1}{p}\right)+\frac{1}{p}$. By Remark 3.6, $\operatorname{deg}\left(v_{1}\right)=\operatorname{deg}\left(w_{1}\right)=\gamma$. From $(2.16),(2.18)$ and $\operatorname{deg}\left(v_{2 m+1}\right)=\operatorname{deg}\left(w_{2 n+1}\right)$, we obtain $m=2 n$. Using that, for $z_{0}>0$, from (5.71), we get

$$
(2 n+1)(2 p+2 a+4 a n-1)=-4 p n(n+1) .
$$

For $(m, n)=(0,0)$, we have a $D(1)$-quadruple $\mathcal{D}_{p}$ from [18], whose elements are not from $\mathbb{R}[X]$. For $n>0$, the right hand side in (5.72) is $<0$, so we conclude that 4 an $<1-2 x_{0}<-1$, which is not possible. For $z_{0}<0$, from (5.71), we get

$$
-4 n^{2}(2 a+p)=2 n+1,
$$

where the left hand side is $\leq 0$ and the right hand side is $>0$, a contradiction. 
CASE 4.C) $v_{2 m+1}=w_{2 n+1}, z_{0}= \pm t, z_{1}= \pm s$ and $\gamma \geq \alpha+2 \beta$.

By (2.11), (2.12) and Remark 2.2, $x_{0}=r, y_{1}=r$ and we have $z_{0} z_{1}>0$. Similarly as in [13], we obtain

$$
\pm 2 \operatorname{astm}(m+1)+2 m r \equiv \pm 2 b \operatorname{stn}(n+1)+2 n r(\bmod c)
$$

and

$$
m(\alpha+\gamma)=n(\beta+\gamma)
$$

In this case $\beta<\gamma$. For $\alpha=\beta$, from (5.73) and (5.74), we obtain $\pm 2 m(m+$ $1)(a-b) \equiv 0(\bmod c)$. This is possible only for $(m, n)=(0,0)$, where $z=$ $v_{1}=w_{1}$, i.e. $d=d_{ \pm}$.

Let $\alpha<\beta<\gamma$. By multiplying (5.73) by st and using (5.1), we get

$$
\pm 2 a m(m+1) \mp 2 b n(n+1) \equiv\left(a+b-d_{-}\right)(n-m)(\bmod c) .
$$

Since $\operatorname{deg}\left(d_{-}\right)<\gamma$, the congruence (5.75) becomes an equation. In this case $\operatorname{deg}\left(d_{-}\right) \geq \beta$. For $\operatorname{deg}\left(d_{-}\right)>\beta$, by considering the leading coefficients of polynomials in obtained equation, we get $m=n$. Thus, by (5.74), $\alpha=\beta$, which is not possible. Therefore, $\gamma=\alpha+2 \beta$. We apply Lemma 3.7. If $d_{-}=a+b \pm 2 r$ then, by considering the leading coefficients of polynomials on both sides of the equation obtained from (5.75), we conclude that $\pm n(n+1)=$ 0 . Therefore, $(m, n)=(0,0)$, which leads to $d=d_{ \pm}$. If $d_{-}=b \pm 2 r^{2} \frac{\sqrt{D_{-}}}{\sqrt{B}}$, similarly, we conclude that $\mp 2 n(n+1)= \pm 2 a \frac{\sqrt{D_{-}}}{\sqrt{B}}(n-m)$ and $\pm 2 a m(m+1)=$ $a \pm 2 \frac{\sqrt{D_{-}}}{\sqrt{B}}(n-m)$, where the signs on the right hand sides of that equations do not depend on the signs on the left hand sides, but are the same in both equations. From that, we obtain $2 a^{2} m(m+1)=a^{2}-2 n(n+1)$ or $-2 a^{2} m(m+$ $1)=a^{2}+2 n(n+1)$. In the first case $2 n(n+1)=a^{2}(1-2 m(m+1))<0$, which is not possible. In the second equation, on the left hand side we have a real number $\leq 0$, and on the right hand side we have a positive real number, which again is not possible.

\section{ACKNOWLEDGEMENTS}

The authors would like to thank Professor Andrej Dujella for his generous help during the preparation of this manuscript. Both authors were supported by the Croatian Science Foundation under the project no. 6422. The second author was also supported by the University of Rijeka research grant no. 13.14.1.2.02.

\section{REFERENCES}

[1] J. Arkin, V. E. Hoggatt and E. G. Strauss, On Euler's solution of a problem of Diophantus, Fibonacci Quart. 17 (1979), 333-339.

[2] A. Baker and H. Davenport, The equations $3 x^{2}-2=y^{2}$ and $8 x^{2}-7=z^{2}$, Quart. J. Math. Oxford Ser. (2) 20 (1969), 129-137.

[3] E. Brown, Sets in which $x y+k$ is always a square, Math. Comp. 45 (1985), 613-620. 
[4] Y. Bugeaud, A. Dujella and M. Mignotte, On the family of Diophantine triples $\{k-$ $\left.1, k+1,16 k^{3}-4 k\right\}$, Glasg. Math. J. 49 (2007), 333-344.

[5] Diophantus of Alexandria, Arithmetics and the book of polygonal numbers, (I. G. Bashmakova, Ed.) (Nauka 1974), 85-86, 215-217.

[6] A. Dujella, Generalization of a problem of Diophantus, Acta Arith. 65 (1993), 15-27.

[7] A. Dujella, The problem of the extension of a parametric family of Diophantine triples, Publ. Math. Debrecen 51 (1997), 311-322.

[8] A. Dujella, On the exceptional set in the problem of Diophantus and Davenport, in: Application of Fibonacci Numbers, Vol. 7 eds. G. E. Bergum, A. N. Philippou, A. F. Horadam, Kluwer, Dordrecht, 1998, pp. 69-76.

[9] A. Dujella, On the size of Diophantine m-tuples, Math. Proc. Cambridge Philos. Soc. 132 (2002), 23-33.

[10] A. Dujella, Bounds for the size of sets with the property $D(n)$, Glas. Mat. Ser. III 39 (2004), 199-205.

[11] A. Dujella, There are only finitely many Diophantine quintuples, J. Reine Angew. Math. 566 (2004), 183-214.

[12] A. Dujella and C. Fuchs, A polynomial variant of a problem of Diophantus and Euler, Rocky Mountain J. Math. 33 (2003), 797-811.

[13] A. Dujella and C. Fuchs, Complete solution of the polynomial version of a problem of Diophantus, J. Number Theory 106 (2004), 326-344.

[14] A. Dujella and C. Fuchs, On a problem of Diophantus for rationals, J. Number Theory 132 (2012), 2075-2083.

[15] A. Dujella, C. Fuchs, F. Luca, A polynomial variant of a problem of Diophantus for pure powers, Int. J. Number Theory 4 (2008), 57-71.

[16] A. Dujella, C. Fuchs and R. F. Tichy, Diophantine m-tuples for linear polynomials, Period. Math. Hungar. 45 (2002), 21-33.

[17] A. Dujella, C. Fuchs and G. Walsh, Diophantine m-tuples for linear polynomials. II. Equal degrees, J. Number Theory, 120 (2006), 213-228.

[18] A. Dujella and A. Jurasić, On the size of sets in a polynomial variant of a problem of Diophantus, Int. J. Number Theory, 6 (2010), 1449-1471.

[19] A. Dujella and M. Kazalicki, More on Diophantine sextuples, in: Number Theory Diophantine problems, uniform distribution and applications, Festschrift in honour of Robert F. Tichy's 60th birthday, eds. C. Elsholtz, P. Grabner, Springer-Verlag, Berlin, 2017, 227-235.

[20] A. Dujella, M. Kazalicki, M. Mikić and M. Szikszai, There are infinitely many rational Diophantine sextuples, Int. Math. Res. Not. IMRN 2017 (2) (2017), 490-508.

[21] A. Dujella and F. Luca, On a problem of Diophantus with polynomials, Rocky Mountain J. Math. 37 (2007), 131-157.

[22] A. Dujella and A. Pethö, A generalization of a theorem of Baker and Davenport, Quart. J. Math. Oxford Ser. (2) 49 (1998), 291-306.

[23] A. Filipin and A. Jurasić, On the size of Diophantine m-tuples for linear polynomials, Miskolc Math. Notes, 17 (2016), 2; 861-876.

[24] Y. Fujita, The extensibility of Diophantine pairs $\{k-1, k+1\}$, J. Number Theory 128 (2008), 322-353.

[25] P. Gibbs, A generalised Stern-Brocot tree from regular Diophantine quadruples. (Preprint). math.NT/9903035.

[26] P. Gibbs, Some rational sextuples, Glas. Mat. Ser. III 41 (2006), 195-203.

[27] B. He, A. Togbé and V. Ziegler, There is no Diophantine quintuple, Trans. Amer. Math. Soc. 371 (2019), 6665-6709.

[28] B. W. Jones, A second variation of a problem of Davenport and Diophantus, Fibonacci Quart. 16 (1977), 155-165. 
[29] B. W. Jones, A variation of a problem of Davenport and Diophantus, Quart. J. Math. Oxford Ser. (2) 27 (1976), 349-353.

[30] A. Jurasić, Diophantine m-tuples for quadratic polynomials, Glas. Mat. Ser. III, 46 (2011), 283-309.

A. Filipin

Faculty of Civil Engineering

University of Zagreb

Fra Andrije Kačića-Miošića 26, 10000 Zagreb

Croatia

E-mail: filipin@grad.hr

A. Jurasić

Department of Mathematics

University of Rijeka

Radmile Matejčić 2, 51000 Rijeka

Croatia

E-mail: ajurasic@math.uniri.hr

Received: 15.3.2018.

Revised: 1.1.2019. 